\title{
1 Metabolic homeostasis controls the diversity of cooperative swarming in
}

\section{2 pathogenic bacteria}

3 Guillem Santamaria ${ }^{1,3+}$, Chen $\mathrm{Liao}^{1+}, \mathrm{Zhe} \mathrm{Wang}^{2}$, Kyu Rhee ${ }^{2}$, Francisco Pinto ${ }^{3}$, Jinyuan

4 Yan $^{1 *}$, Joao B. Xavier ${ }^{1 *}$

$5 \quad{ }^{1}$ Program for Computational and Systems Biology, Memorial Sloan-Kettering Cancer Center,

6 New York, NY, USA

$7 \quad{ }^{2}$ Department of Medicine, Weill Cornell Medical College, New York, NY

$8 \quad{ }^{3}$ BioISI - Biosystems \& Integrative Sciences Institute, Faculty of Sciences, University of

9 Lisboa, Lisboa, Portugal

10

$11+$ these authors contributed equally

$12{ }^{*}$ Correspondence: yanj2@mskcc.org and xavierj@mskcc.org 


\section{Abstract}

15 Many bacteria have an incredible ability to swarm cooperatively over surfaces. But swarming

16 phenotypes can be quite different even between strains of the same species. What drives this

17 diversity? We compared the metabolomes of 29 clinical Pseudomonas aeruginosa isolates

with a range of swarming phenotypes. We identified that isolates incapable of secreting rhamnolipids — a surfactant needed for swarming — had perturbed tricarboxylic acid (TCA) cycle and amino acid pathways and grew exponentially slower in glycerol minimal medium.

21 Analysis of the metabolome signatures and simulations using a genome-scale model led to a mechanism which joins these observations: Strains subject to higher oxidative stress levels grow slower and shut down rhamnolipids secretion, a carbon overflow mechanism possibly to direct carbon resources towards costly stress response pathways to maintain cell viability. In vitro experiments confirmed that rhamnolipid non-producers deal worse with oxidative stress, linking intracellular redox homeostasis - a individual-level trait - to swarming-a population-level behavior. This mechanism helps explain the metabolic constraints on bacteria when secreting byproducts to interact with others-competitively and cooperatively-in microbial communities.

\section{Significance}

31 Swarming motility has been associated with virulence of many human bacterial pathogens.

32 The pathogen Pseudomonas aeruginosa swarms by cooperatively secreting surfactants called 33 rhamnolipids to lubricate surfaces. To understand why some $P$. aeruginosa strains swarm and 34 others do not, we combined metabolomics, computational modeling and in vitro experiments 35 to study the different swarming behaviors of 29 isolates of Pseudomonas aeruginosa obtained 36 from infected patients. We found that strains can only produce rhamnolipids if they can

37 maintain redox homeostasis. We propose that single cells must have low internal redox stress 
38 levels before they can produce rhamnolipids, which work as an overflow of carbon

39 metabolism into a cooperative secretion that brings a fitness benefit to the entire swarming

40 population. This mechanism links single-cell physiology and a population-level cooperative

41 behavior key to the fitness and virulence of $P$. aeruginosa, a major source of hospital

42 acquired infections.

\section{Synopsis}

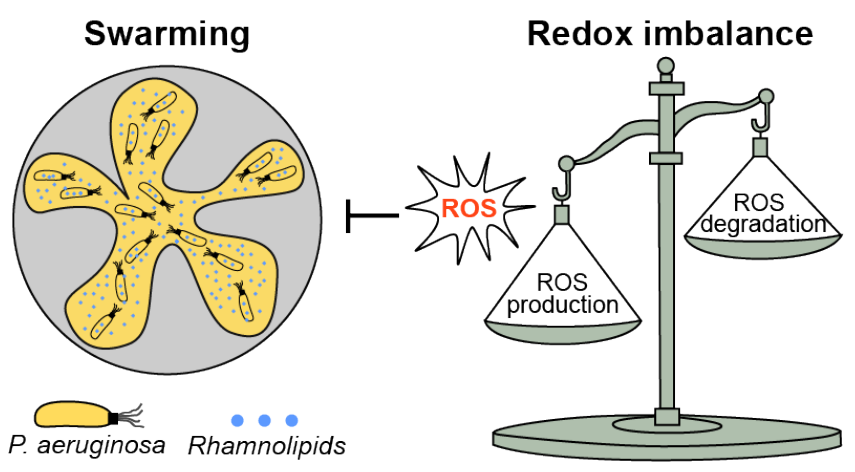

This study combined metabolomics, computational modeling and experiments to explain the swarming diversity in Pseudomonas aeruginosa, yielding new insights on the genetic and metabolic controls of bacterial swarming behavior

- Rhamnolipid secretion is necessary, but insufficient, for swarming

- P. aeruginosa strains unable to produce rhamnolipids in the glycerol minimal medium have perturbed metabolism, slow growth and elevated oxidative stress

- Rhamnolipid producers cannot produce rhamnolipids in succinate as the sole carbon source which causes greater ROS (reactive oxygen species) production

- The requirement of redox homeostasis for rhamnolipid production ensures that the overflow of precious carbon for population-level benefit is prudently controlled

- This study helps linking single cell physiology with collective behavior key to the fitness and virulence of a pathogenic bacterium. 


\section{Introduction}

58 Swarming enables millions of bacteria to travel together across centimeter-long distances in a

59 few hours (Deforet et al, 2014). This cooperative trait found typically in the phyla Alpha-

60 proteobacteria and Gamma-proteobacteria is a complex phenotype that requires the coordinated expression of many genes: Swarming requires flagella or pili to propel the cells, cell-cell interactions to maintain population integrity during migration, and the collective secretion of biosurfactants to lubricate the surface (Kearns, 2010). Studies over the past two decades revealed many molecular details of swarming motility through genetic (e.g., knockouts, transgenesis) and environmental (e.g., nutrient composition and viscosity of culture medium) perturbations (Köhler et al, 2000; Mattingly et al, 2018) as well as through experimental evolution (van Ditmarsch et al, 2013). Some genes that modulate swarming impact key cellular activities including tricarboxylic acid (TCA) cycle and stress response (Inoue et al, 2007; Tremblay \& Déziel, 2010) and therefore have pleiotropic effects. Despite these insights, we lack a systems-level understanding of this cooperative bacterial behavior (Kim \& Surette, 2004; Inoue et al, 2007).

Pseudomonas aeruginosa - an opportunistic human pathogen and a major cause of hospitalacquired infections (Klevens et al, 2007) — has a remarkable swarming ability that produces long straight segments (tendrils) in its fractal-like swarming pattern. Similar to other swarming species, $P$. aeruginosa requires both flagella and pili to move, the LasR-LasI and RhlR-RhlI quorum sensing systems to communicate within population, and the ability to produce rhamnolipids to lubricate the surface (Köhler et al, 2000). Rhamnolipids are a mixture of biosurfactants that consist of 3-(3-hydroxyalkanoyloxy) alkanoic acids (HAAs), mono-rhamnolipids and di-rhamnolipids (Abdel-Mawgoud et al, 2010). RhlA is the only enzyme required to drive the conversion of fatty acid biosynthesis intermediates ( $\beta$ - 
hydroxyacyl-ACP) to HAAs (Zhu \& Rock, 2008), whereas RhlB and RhlC each add one molecule of rhamnose to the HAAs to produce mono-rhamnolipids and di-rhamnolipids in sequential steps (Chong \& Li, 2017). The genes encoding RhlA and RhlB are located in the same operon $\operatorname{rhlAB}$, which is regulated by the quorum-sensing cascade headed by LasR-LasI and followed by RhlR-RhlI (Medina et al, 2003). Other than quorum-sensing signals, rhlAB expression is also controlled by nutrient cues such as an excess of carbon source (Xavier et al, 2011; Boyle et al, 2015; Mellbye \& Schuster, 2014). The combination of quorum-sensing and metabolic signals is called metabolic prudence; it prevents cheating by rhamnolipid nonproducers such as the $\Delta r h l A$ mutant because it prudently ensures that rhamnolipids are only produced at the right time when the production benefits outweigh its costs. Strains that produce rhamnolipids too soon or overproduce rhamnolipids, such as a strain engineered with the inducible $\mathrm{P}_{\mathrm{BAD}-r h l A}$ construct, have less carbon available for growth and are susceptible to cheating by non-producers (Xavier et al, 2011; de Vargas Roditi et al, 2013).

Besides the role of metabolic prudence, which regulates rhamnolipid production and makes swarming robust to cheating, other aspects of metabolism might have influenced the evolution of swarming across the $P$. aeruginosa phylogenetic tree. Metabolism is the currency of all physiological processes that support life (Smith \& Morowitz, 2004) and a major determinant of social behavior (Biro \& Stamps, 2010). A previous study (Boyle et al, 2017) compared the intracellular metabolites of the laboratory strain PA14 and several mutants of that strain with different swarming phenotypes. The $\Delta r h l A$ mutant can neither swarm nor produce rhamnolipids, but it had less than 10 metabolites perturbed compared to the wild-type. The $\triangle c b r A$ mutant, which lacks a global regulator of carbon-nitrogen balance, had impaired swarming and lower growth rate with dozens of perturbed metabolites. Notably, the $\triangle c b r A$ mutant still had the flagella needed to move and secreted even more rhamnolipids than the wild-type. More interestingly, its swarming phenotype could be rescued by 
compensatory point mutations in the RNA chaperone $h f q$ through rewiring of metabolic

107 network to reconstruct a unique metabolome which was distinct from both the $\Delta c b r A$ and the

108 wild-type (Boyle et al, 2017).

109 Here we leveraged a collection of 29 clinical isolates of $P$. aeruginosa that we previously

110 isolated from hospitalized cancer patients in Memorial Sloan-Kettering Cancer Center

111 (MSKCC) (Yan et al, 2017). These strains span a wide range of swarming phenotypes;

112 however, they evolved independently in environments that are largely unknown to us and

113 exhibit great genetic diversity, which makes it hard to pin down the exact genes responsible

114 for their different swarming behaviors. Since the differences cannot be solely explained by

115 genetics, here, in this study, we combined genomics with metabolomics, growth curve

116 analysis and computational models to study the metabolic differences underlying the

117 observed swarming diversity. Swarming requires rhamnolipid production; here we found that

118 rhamnolipid-producing strains have reduced TCA cycle activity, increased levels of amino

119 acids, and slower exponential-phase growth rate compared to the non-producing strains. We

120 further provided both computational and experimental evidences showing that these

121 metabolic changes are likely caused by a higher oxidative stress. This finding suggests that

122 rhamnolipid production is a form of overflow metabolism that is only activated in strains with

123 sufficient capability to detoxify reactive oxygen species (ROS) produced by energy

124 metabolism and maintain redox balance. This mechanism links redox homeostasis with

125 swarming cooperation, highlighting the importance of metabolism in the evolution of a social

126 behavior.

\section{Results}

128 Phylogenetic analysis suggests loss of rhamnolipid production drove loss of swarming in

129 some isolates. We started by analyzing the shapes of swarming colonies of clinical isolates 
130 and three common reference strains whose genomes vary widely (Fig. 1). We used image

131 analysis to measure six morphological features of the colonies, including perimeter,

132 maximum length, area, circularity, skeleton longitude and eccentricity (see Materials and

133 methods for their definitions). A principal component analysis of these morphological

134 features revealed that the maximum length and circularity captured the most variability of the

135 shape diversity in our tested strains (Supplementary Fig. 1). We quantified the swarming

136 ability of all strains using a linear combination of the two features but found no significant

137 correlation of the swarming scores with the phylogeny inferred from their core genomes

138 (Moran's I test, p-value=0.93). Since production of rhamnolipids is required for swarming

139 motility, we measured the ability of strains to produce rhamnolipids in a synthetic media with

140 glycerol as the sole carbon source (Boyle et al, 2015). As expected, all strains able to swarm

141 produced rhamnolipids. Not all rhamnolipid producers swarmed, though, which confirms that

142 rhamnolipids are necessary but not sufficient for swarming (Caiazza et al, 2005; Déziel et al,

143 2003). Similar to swarming phenotype, the ability to produce rhamnolipids, semi-

144 quantitatively classified as non-, mild- and strong-producers (Materials and Methods), was

145 also uncorrelated with the phylogeny (Fig. 1B, Moran's I test, p-value=0.14). To better assess

146 the link between swarming and rhamnolipid production, we built an evolutionary model to

147 reconstruct their ancestral states along the phylogenetic tree. The model predicted that the

148 common ancestor of all the strains can both swarm and produce rhamnolipids. This prediction

149 suggests that the diversity of swarming found across the isolates was caused by some strains

150 losing their ability to swarm or produce rhamnolipids, not by concurrent lineages evolving

151 those functions independently. The model also suggests that rhamnolipid production is more

152 conserved than swarming: the immediate ancestors of all non-producers are inferred to be

153 rhamnolipid producers (Supplementary Figure 2A). By contrast, the loss of swarming

154 occurred earlier than that of rhamnolipid production in several lineages (Supplementary 
155 Figure 2B), a result which is also expected because swarming depends on other factors

156 besides rhamnolipid production (Kearns, 2010).

157 The loss of rhamnolipid production is partially explained by the absence of genes. Since

158 the phylogeny inferred from core genes was unable to explain the observed diversity of

159 rhamnolipid production, we asked whether accessory genes, particularly those only missing

160 in rhamnolipid non-producers, could explain the diversity. Here we focused on the

161 comparison between rhamnolipid producers (both mild and strong rhamnolipid-producing

162 strains; the same below) and non-producers, rather than between swarmers and non-

163 swarmers, because (1) the latter phenotype is harder to determine non-arbitrarily and (2)

164 rhamnolipid production is itself a predictor of swarming (all rhamnolipid non-producers lose

165 swarming). We then compared the genomic sequences between the 8 rhamnolipid non-

166 producers and the remaining producers using reciprocal blast to explore the genetic features

167 that may be associated with rhamnolipid production. We identified 304 genes

168 (Supplementary File 1) only absent in those non-producers that may directly contribute to the

169 loss of rhamnolipid production (89 well annotated genes are listed in Table 1): F5677 lacks

170 the flagella motor switch protein fliM, and $q s c R$ - a quorum-sensing control repressor;

171 W36662 lacks the type IV pili assembly protein pilC and its sensor kinase pilS; and F63912,

172 W36662, W60856 lack three quorum-sensing genes las $R$, rhlR, and rhlI. It was previously

173 shown that the single-gene knockout mutants $\Delta f l i M, \Delta p i l S, \Delta r h l R$ and $\Delta r h l I$ (Köhler et al,

174 2000; Overhage et al, 2007; Kamatkar \& Shrout, 2011), but not $\Delta$ pilC (Luo et al, 2015),

175 displayed strong swarming-deficient phenotypes. The mutant of a two-component response

176 regulator gene pirR that is missing in $\mathrm{H} 27930$ was found to repress swarming in multiple

177 culture media (Kollaran et al, 2019). S86968 lacks $n f x B$, whose mutation caused global

178 dysregulation of physiology and metabolism in P. aeruginosa including impaired swarming

179 (Stickland et al, 2010). Notably, genes encoding rhamnolipid biosynthsis (rhlA, rhlB, rhlC) 
are intact and contain no mutation in all non-producers, indicating that the mutations that lead to the loss of rhamnolipid production are linked to the regulation of the pathway, either in other regulatory genes (in coding or non coding regions) or in non-coding regions of the biosynthetic genes.

184 Besides these functions that are directly linked to rhamnolipid production and swarming, 185 genes involved in carbon catabolism, biofilm, chemotaxis, antibiotic resistance, virulence and redox regulation were also found missing in rhamnolipid non-producers. M55212 misses the catabolite repression control gene $\operatorname{crc}$, although a $\operatorname{crc}$ transposon mutant is able to swarm as well as the wild-type (Yeung et al, 2011). H27930 lacks the methyl-accepting chemotaxis genes $p c t A$ and $p c t B$ and the biofilm biosynthesis gene pelA. Both M1608 and S86968 lack

190 the pyridoxal phosphate (vitamin $\mathrm{B}_{6}$ ) biosynthetic gene $p d x A$ and the hcnABC genes coding 191 for the virulence factors hydrogen cyanide (Pessi \& Haas, 2000). F5677 cannot express PhzA2 for biosynthesis of phenazine - a well-known virulence factor. The absence of amrB that encodes a membrane protein of the AmrAB-mediated efflux system in M1608 suggests

194 that the strain may be sensitive to the aminoglycoside antibiotic (Westbrock-Wadman et al, 195 1999). The missing genes for redox regulation include soxR (absent in F5677) — a redox sensitive transcriptional regulator, $k a t E$ (absent in M1608) - a catalase that degrades $\mathrm{H}_{2} \mathrm{O}_{2}$

197 (Mulvey et al, 1988), and gor (absent in M1608) - a flavoprotein that oxidizes glutathione, which plays an important role in protection against $\mathrm{H}_{2} \mathrm{O}_{2}$ damage (Perry et al, 1991).

199 Rhamnolipid producers grow faster in glycerol. The missing genes in the 8 non-

200 rhamnolipid-producers (Table 1) include several global transcriptional regulators (e.g., rhlR, 201 lasR, crc, $n f x B$, soxR) that are broadly important for cell metabolism and growth. Their absence may cause growth-independent or -dependent metabolic effects on rhamnolipid production and swarming. We first tested whether growth is a predictor for the observed 
diversity. We monitored growth curves of all our strains over $48 \mathrm{~h}$ in the same glycerol media

that we had used to measure rhamnolipid production. These growth curves exhibited observed among 6 out of 9 swarming strains (Supplementary Fig. 3). However, some rhamnolipid producers clustered with non-producers, suggesting that rhamnolipid production cannot be classified by the entire growth curves and more sophisticated methods to extract local growth curve features were needed.

211 We then used both unsupervised and supervised learning approaches to identify features in

212 the growth curves associated with rhamnolipid production. For the unsupervised approach, 213 we used non-negative matrix factorization (Lee \& Seung, 1999) which decomposed each 214 growth curve as a weighted sum of three basis functions (i.e., features). Although the growth curves of the rhamnolipid producers (orange lines) and non-producers (blue lines) largely overlapped (Fig. 2A), there was a significant difference in the weights associated with basis 1

217 between the two groups (Fig. 2B), which suggests a link between rhamnolipid production and

218 bacterial growth. To pinpoint features associated with rhamnolipid production, we divided 219 each growth curve into three phases based on its shape (Fig. 2C and Supplementary Fig. 4) and defined 7 quantitative features to characterize each growth phase (Fig. 2D and Supplementary File 2). Using Random Forest classification, we found that the top two features with the highest explanatory power were the maximum and averaged specific growth rates in phase I when growth speeds up (Fig. 2E). Considering the majority of rhamnolipids 224 are produced in phase II when growth slows down (Boyle et al, 2015), our finding revealed a strong temporal link between exponential growth rate in phase I and rhamnolipid production in phase II. We hypothesized that the link might be mediated by metabolic homeostasis: its

227 disruption could slow down exponential growth in phase I and disfavor rhamnolipid secretion 
in phase II by saving precious carbon resources for cell maintenance and stress responses under metabolic imbalanced conditions.

\section{Rhamnolipid non-producers have perturbed TCA cycle and amino acid pathways.}

231 Similar to our growth curve analysis, we adopted both unsupervised and supervised learning 232 approaches to test the associations of intracellular metabolite levels with swarming and 233 rhamnolipid production. We profiled the intracellular metabolome of all our strains in the same glycerol minimal medium (except for two rhamnolipid non-producers M55212 and F23197 which grew too slow) during the transition between phase I and phase II when chromatography-Mass spectrometry) we identified a total of 92 compounds spanning a wide range of abundances (Supplementary Fig. 5A), including 67 metabolites whose identities are known and abundances vary significantly across all the tested strains (Fig. 3A). After data normalization and imputation (Supplementary Fig. 5B), hierarchical clustering showed the consistency of data across all three replicates for each strain (Fig. 3B), except for one replicate of H47921 (sample 25, an outlier removed from further analysis). The similarity among the metabolomics data yielded three major clusters with one cluster (middle cluster in

244 Fig. 3B) containing all strong swarmers (strain name in red) and only rhamnolipid producers.

245 However, no clear metabolic pattern was found among non-producers: Two non-producers

246 were grouped in the left cluster and 4 in the right cluster, and they all had metabolomics 247 profiles similar to producers within the same cluster.

248 The lack of a clear metabolomic profile for rhamnolipid non-producers led us to search for 249 specific differential metabolites using supervised methods. We fitted our metabolomic data 250 (explanatory variables) to categorized rhamnolipid production (response variable) using 251 Orthogonal Projections to Latent Structures-Discriminant Analysis (OPLS-DA) (Cloarec et 
$a l, 2005)$. The OPLS-DA produced a reasonable fit $\left(\mathrm{R}^{2}=0.82, \mathrm{Q}^{2}=0.66, p\right.$-value $\left.=5 \mathrm{e}-4\right)$

with the single predictor that separated rhamnolipid non-producers from producers explaining

explained by three orthogonal components that capture variation within both groups, again

showing that there is a high overlap between the metabolomic profiles of rhamnolipid

producers and non-producers. The loading values of the predictive component reflect how

much each compound contributes to the separation between rhamnolipid producers and non-

(Fig. 4B and Supplementary Fig. 6).

We then used a univariate Mann-Whitney $U$ test to identify the metabolites differentially abundant in producers and non-producers. We found 24 such metabolites (bars with black outline in Supplementary Fig. 6) and these metabolites were then used to identify pathways perturbed in non-producers using the FELLA algorithm (Picart-Armada et al, 2017, 2018) acid metabolism (Fig. 4B). 3 out of 6 metabolites in the TCA cycle-fumarate, succinate and citrate - were significantly changed: Fumarate and malate showed a positive loading value, while citrate, succinate, and to a less extent cis-aconitate and alpha-ketoglutarate were negative (Fig. 4B). Pyruvate remained relatively constant across all the strains (Fig. 3A), implying that the differential responses in the TCA cycle were independent from the changes in its upstream central carbon metabolism.

272 Besides the TCA cycle metabolites, the majority of annotated compounds in the metabolism 273 of branched chain amino acids (leucine/isoleucine, valine) and sulfur-containing amino acids 274 (cysteine/methionine) had higher abundance in rhamnolipid non-producers relative to producers. In fact, with the exception of glutamate, proline and tryptophan, all other amino 
acids detected in our metabolomics data (threonine, alanine, aspartate, glutamine, cysteine,

277 lysine, histidine, tyrosine, and arginine) were higher in non-producers (Supplementary Fig.

278 6). A striking exception to this trend was formylmethionine (fMet), which had lower 279 abundance in non-producers. Diminished level of fMet had also been found in the $\Delta r h l A$ 280 mutant of $P$. aeruginosa (Supplementary Fig. 7) (Boyle et al, 2017). Since $\Delta r h l A$ mutant 281 grows with a similar growth rate as the wild-type (van Ditmarsch \& Xavier, 2011) the link between fMet and rhamnolipid production should not be simply due to a growth defect.

Metabolome signature and computer simulations link high oxidative stress to lower

rhamnolipid production. So far our metabolomics data show that the non-producers have perturbed TCA cycle activity and grow slowly. As shown in Fig. 4C, the TCA cycle harbors

286 five enzymes with Fe-S clusters (aconitase A, aconitase B, succinate dehydrogenase, fumarase A, fumarase B (Py \& Barras, 2010)) and thus represents one of the most vulnerable pathways subject to attacks of ROS. It is likely that higher oxidative stress in the nonproducers reduces flux through the TCA cycle, which would explain their slower growth. The significantly opposite association of succinate and fumarate we found in rhamnolipid production indicates the reduced activity of succinate dehydrogenase (SDH) under oxidative stress conditions. SDH is a membrane-bound dehydrogenase linked to the respiratory chaina major site of ROS production in the cell-and also a member of the TCA cycle that contains [2Fe-2S], [3Fe-4S], and [4Fe-4S] clusters (Ayala-Castro et al, 2008), ROS that damages Fe-S clusters could decrease SDH activity in vivo. The metabolomics data also

297 showed that some intermediate metabolites in amino acid biosynthetic pathways, which are also substrates of Fe-S containing enzymes, accumulated in the non-producers (Fig. 4C). For example, the glutamate synthetase that consists of a large chain (encoded by gltB) and a small chain (encoded by gltD). Both subunits contain Fe-S clusters and catalyze the production of 
301 glutamate from oxoglutarate and glutamine. We found slightly higher oxoglutarate and

302 glutamine as well as lower glutamate in the non-producers. These observations collectively

303 suggest that the rhamnolipid non-producers might have higher oxidative stress levels than the

304 producers.

305 These reasons suggest that the cause for impaired rhamnolipid production might be that non-

306 producing strains suffer from high oxidative stress. We then sought to use a whole-genome

307 reconstruction model to study how oxidative stress could lower the flux of carbon available to

308 rhamnolipid production. We modified a high-quality genome-scale model of Pseudomonas

309 metabolism, iJN1411 (Nogales et al, 2017, 2020), to simulate growth in the culture medium

310 used in our metabolomics experiments with glycerol and ammonium as the sole carbon and

311 nitrogen sources. Rhamnolipids are produced when carbon is in excess (Boyle et al, 2015). In

312 this model rhamnolipids secretion occurs when the carbon-to-nitrogen $(\mathrm{C}: \mathrm{N})$ ratio exceeds a

3136.3 threshold (Supplementary Fig. 8). We set C:N to 10.0, which exceeds the minimum

314 threshold, and we varied the redox stress level by changing the flux levels of three redox

315 molecules: NADH (reduced nicotinamide adenine dinucleotide), NADPH (reduced

316 nicotinamide adenine dinucleotide phosphate) and GSH (reduced glutathione); these

317 molecules are responsible for the bulk of cellular electron transfer and likely to be the main

318 sources of ROS (Xiao \& Loscalzo, 2019). Although redox stress is ultimately caused by high

319 ROS levels, not fluxes, and ROS was not an explicit variable in our model, we assumed that

320 ROS can perturb intracellular metabolic fluxes, particularly the fluxes of redox molecules,

321 and asked how cell growth and rhamnolipid secretion respond to these ROS-mediated

322 indirect perturbations.

323 For all three redox molecules, we found that the maximum growth rate was maintained at an

324 intermediate flux range (redox homeostasis, white area) while any flux rate outside the range 
325 (gray shading) gradually reduced growth rate (Fig. 5, upper panels). What generally

326 accompanied the compromised growth rate was the abrupt shutdown of secretion of

327 rhamnolipid precursors (HAA), mono- and di-rhamnolipids as well as many (but not all)

328 central carbon metabolites, except for extremely small GSH flux (Fig. 5, lower panels).

329 Importantly, reducing any of these secretion fluxes did not reduce the growth rate, nor did it

330 provide growth benefits (i.e., each individual flux could be as low as zero without reducing

331 maximum growth rate). This observation agrees with the data that the $\triangle r h l A$ mutant of $P$.

332 aeruginosa type strain PA14 has the same grow rate as its wild-type strain in the same

333 glycerol minimal medium (van Ditmarsch \& Xavier, 2011). Notably, most of the overflowed

334 carbon-rich molecules, including HAA, mono- and di-rhamnolipids, were only secreted when

335 the growth rate maintained its maximum value under excess carbon. The computational

336 inference that rhamnolipids, as well as other central carbon metabolism intermediates, can be

337 secreted under maximum growth suggests that $P$. aeruginosa cells have the flexibility to

338 overflow carbon through alternative metabolic pathways without compromising growth. The

339 fact that $P$. aeruginosa maintains the secretion of rhamnolipids among these options supports

340 that rhamnolipid secretion was favored by natural selection for reasons other than improving

341 single-cell fitness. Collectively, these simulations support a link between rhamnolipids

342 secretion and redox stress, where fast growth and rhamnolipid secretion are both

343 metabolically constrained by cellular redox homeostasis.

344 Experiments support the link between growth, oxidative stress and rhamnolipid

345 production. We validated the link between oxidative stress and rhamnolipid production by

346 two additional experiments. In the first experiment, we monitored growth and rhamnolipid

347 production of all our clinical isolates when they grew in the same minimal medium but with

348 succinate as the sole carbon source (Supplementary Fig. 9). Since succinate enters the carbon

349 metabolism directly through the TCA cycle, we expected that, compared to growth in 
glycerol, the initial TCA cycle flux would be much faster and generates higher ROS level that impairs the sustained cell growth and rhamnolipid secretion. Indeed, none of the strains that produced rhamnolipids in glycerol produced rhamnolipids in succinate. We similarly extracted 7 phase-dependent growth curve features of our strains grown in succinate (Supplementary File 3) and compared their values to the same features measured in glycerol (Supplementary Fig. 10). The comparison showed that all strains had shorter lag phases and grew exponentially faster in succinate. The strains reached much lower cell densities even though the succinate medium had the same initial molar concentration of carbon as the glycerol medium. More interestingly, phase II was almost absent in succinate compared to glycerol. The strains all switched abruptly from phase I to phase III, suggesting that cells might have accumulated more oxidative stress during phase I, which caused a sudden stop in growth as well as carbon overflow.

In the second experiment, we monitored the hydrogen peroxide $\left(\mathrm{H}_{2} \mathrm{O}_{2}\right)$ level when growing our Pseudomonas strains in the glycerol minimal medium (Fig. 6A,B). $\mathrm{H}_{2} \mathrm{O}_{2}$ is a representative ROS that can diffuse freely between cell and the environment. By measuring the fluorescent signal intensity produced by the Amplex assay (Materials and Methods), we

366 found a baseline production of $\mathrm{H}_{2} \mathrm{O}_{2}$ in the absence of cells, possibly due to photooxidation of 367 Amplex Red reagent (Zhao et al, 2012). When cells were added, Pseudomonas strains can 368 degrade $\mathrm{H}_{2} \mathrm{O}_{2}$ by producing many antioxidant enzymes including catalases, glutathione 369 reductase, NADH peroxidase and cysteine-based peroxidases (Mishra \& Imlay, 2012). We 370 computed the signal intensity difference between cell culture and baseline as a proxy of the 371 ability of each strain to remove $\mathrm{H}_{2} \mathrm{O}_{2}$ and broadly combat oxidative stress. We saw that all 372 strains except for M1608 quenched the signal over the first 18 hours (Fig. 6B). Most 373 importantly, the non-producers (blue lines) removed less $\mathrm{H}_{2} \mathrm{O}_{2}$ than mild rhamnolipid 374 producers (green lines) and strong producers (red lines), supporting our hypothesis that non- 
producers are worse at removing ROS. Unsurprisingly, the worst strain in this assay was M1608, which lacks katE and gor (Table 1), both of which are important for $\mathrm{H}_{2} \mathrm{O}_{2}$

377 degradation. To factor out the possibility that the non-producers performed worse due to

378 lower cell density, we calculated the $\mathrm{H}_{2} \mathrm{O}_{2}$ removal rate per cell and observed similar relative

379 trend of $\mathrm{H}_{2} \mathrm{O}_{2}$ degradation between non-producers and producers (Fig. 6C). Formally, we used a linear mixed-effect model to test the effect of rhamnolipid production on per-cell $\mathrm{H}_{2} \mathrm{O}_{2}$ removal rate (Materials and Methods). The effect size of mild and strong producers relative to the non-produces are $1.25 \mathrm{x}$ and $1.41 \mathrm{x}$ stronger respectively with both $\mathrm{p}$-values $<0.001$, confirming that rhamnolipid producers deal better with oxidative stress.

\section{Discussion}

385 Our data comparing growth phenotypes and metabolomes posit a model to explain the 386 diversity of swarming phenotypes we saw in P. aeruginosa isolates (Fig. 7): Some strains can

387 produce rhamnolipids because they maintain redox homeostasis due to their greater abilities to degrade ROS. Rhamnolipid non-producers by contrast had disrupted redox homeostasis, causing slower growth with reduced TCA cycle activity and increased amino acid concentration. The impaired ability to degrade ROS by non-producers is possibly caused by

391 loss of genes that encode ROS detoxification functions (e.g., M1608 lacks katE and gor).

392 Consistently, it was reported that OxyR — oxidative stress regulator that controls expression

393 of antioxidant genes - is required for swarming and rhamnolipid production (Vinckx et al, 394 2010).

395 All our clinical strains were isolated from hospitalized patients, a host-associated

396 environment where we may expect redox stresses such as ROS imposed by the immune 397 system in their fight against pathogens (Puertollano et al, 2011) and by antibiotics treatment 398 (Zhu et al, 2019). Non-producers of rhamnolipids seem less capable of dealing with oxidative 
stresses, and it remains unclear what selected for this loss of function in vivo. Rhamnolipid production was, nonetheless, relatively frequent across the $P$. aeruginosa phylogenetic tree.

401 The genes for rhamnolipid biosynthesis $(r h l A, r h l B, r h l C)$ remained intact across all of our 402 clinical isolations, even in non-producers. This suggests that the loss of rhamnolipid 403 production in some strains may have resulted from broader metabolic adaptations such as oxidative stress responses. In support of our model we saw that rhamnolipid producers could quench oxidation faster than non-producers (Fig. 6). Accumulation of oxidative stress due to slow removal can reduce SDH activity and perturb TCA cycle (Fig. 7). Others have shown that $P$. aeruginosa decreases expression of the key quorum-sensing genes (LasI, LasR, RhlI, RhlR, PqsA and PqsR) in response to oxidative stress caused by exposure to $\mathrm{H}_{2} \mathrm{O}_{2}$ (Mohamed et al, 2020). Considering quorum sensing regulates hundreds of genes (Lequette et al, 2006), shutting down the cooperative traits that are under quorum-sensing regulation-including

411 rhamnolipid secretion — could save precious resources for oxidative stress responses.

412 We also saw that rhamnolipid non-producers had significantly lower levels of fMet than 413 producers. Interestingly, this also happens in the $\Delta r h l A$ mutant compared to its wild-type 414 strain PA14 (Boyle et al, 2017). fMet plays a role in translation initiation, and in the quality 415 control mechanisms that degrade misfolded proteins (Piatkov et al, 2015). Bacillus subtills 416 lacking Formyl-Methionine Transferase (FMT) — the enzyme attaching a formyl group to 417 methionine loaded on tRNA ${ }^{\text {fmet }}$ - are more sensitive to $\mathrm{H}_{2} \mathrm{O}_{2}$ and defective in swarming (Cai 418 et al, 2017). The link between fMet, swarming and oxidative stress is intriguing and warrants 419 further research.

420 Only the strains that grew fast on glycerol could produce rhamnolipids in that medium. This 421 supports the notion that rhamnolipid production is a form of overflow metabolism. Overflow 422 metabolism is associated with fast growth such as bacteria under conditions of high glucose 
consumption. It occurs when cells simultaneously operate on both energy-efficient (e.g.,

424 respiration) and -inefficient (e.g., fermentation) pathways and secret metabolic byproducts that could otherwise be used for catabolism or anabolism. This phenomenon has been observed in different systems: aerobic fermentation in Escherichia coli (Farmer \& Jones, 1976), the Crabtree effect in Saccharomyces cerevisiae (De Deken, 1966) and the Warburg effect in cancer cells (Vander Heiden et al, 2009). Many molecular mechanisms have been proposed and validated to account for the seemingly wasteful usage of low energy-yield pathway in presence of a higher-energy-yield alternative (Basan et al, 2015; Szenk et al, 2017). For example, the respiratory flux that would be required for fast growth without

432 fermentation could accumulate NADH that could not be recycled to $\mathrm{NAD}^{+}$by respiration 433 alone, because of a limited surface/volume ratio (Szenk et al, 2017). Overexpression of 434 NADH oxidase in both E. coli (Vemuri et al, 2006) and S. cerevisiae (Vemuri et al, 2007) reduced the NADH/NAD ${ }^{+}$ratio and suppressed overflow metabolism. One key difference between $P$. aeruginosa rhamnolipid production and the overflow metabolism of other systems is that rhamnolipids are controlled by quorum-sensing in addition to nutrient cues (Boyle et al, 2015), which results in a cell-density-dependence.

439 Should rhamnolipid production provide a fitness benefit for $P$. aeruginosa under any 440 environmental conditions? We have previously shown that rhamnolipid production is 441 dispensable for normal growth of $P$. aeruginosa in laboratory conditions: the $\triangle r h l A$ mutant is 442 an engineered rhamnolipid non-producer defector but has almost the same growth rate (van 443 Ditmarsch \& Xavier, 2011) and very similar metabolomic profiles (Boyle et al, 2017) as the 444 wild-type. This is consistent with our flux-balance analysis of Pseudomonas metabolism, 445 where shutting down rhamnolipid secretion did not impact the growth rate because the excess 446 carbon could be alternatively secreted in other forms such as acetate (Fig. 5). Notably, $\Delta r h l A$ 447 had higher intracellular levels of gamma-Glutamylcysteine (Supplementary Fig. 7), the 
immediate precursor of glutathione. Since glutathione is a well-known antioxidant that

449 protects cells from ROS damage (Ezraty et al, 2017), $\Delta r h l A$ may exhibit slight oxidative

450 burden. This could explain why the $\Delta r h l A$ has a marginally lower fitness and fails to invade

451 the wild-type in swarming competitions (de Vargas Roditi et al, 2013; Monaco et al, 2020).

452 Our mechanism proposes that increased oxidative burden may be related to altered redox

ratio $\left(\mathrm{NAD}(\mathrm{P}) \mathrm{H} / \mathrm{NAD}(\mathrm{P})^{+}\right)$, considering that fatty acid biosynthesis that provides precursors

454 for rhamnolipid production regenerates $\mathrm{NAD}(\mathrm{P})+$ from $\mathrm{NAD}(\mathrm{P}) \mathrm{H}$. Notably, the active

455 shutdown of rhamnolipid production in redox imbalanced condition would save precious

456 carbon and enzyme resources to respond to oxidative stress.

457 Our results confirm the role that metabolic byproducts play in linking single-cell physiology 458 and social behavior. Microorganisms secrete various products, including iron scavenging 459 siderophores (Griffin et al, 2004), extracellular enzymes (Altindis et al, 2015), toxins and 460 cell-cell signaling molecules (Schmidt et al, 2019). In synthetic and natural microbial 461 communities the products of one microorganism can impact another, cooperatively or 462 competitively (Xavier, 2011). Our model proposes that the choice of a given molecule to 463 drive a social interaction is not arbitrary: molecules that conciliate individual-level and 464 population-level interests are more likely to drive microbial sociobiology.

\section{Materials and Methods}

466 Media. Glycerol and succinate synthetic media were prepared with $800 \mathrm{ml}$ of Milipore water,

$467200 \mathrm{ml}$ of $5 \mathrm{X}$ minimal salts buffer, $1 \mathrm{ml}$ of $1 \mathrm{M}$ magnesium sulphate, $0.1 \mathrm{ml}$ of calcium 468 sulphate with $0.5 \mathrm{gN} / 1$ nitrogen (ammonium sulphate), iron at $5 \mu \mathrm{M}$ (iron III sulphate) and 3.0

$469 \mathrm{gC} / 1$ glycerol or succinate, respectively. Swarming agar consisted in $800 \mathrm{ml}$ of Milipore water,

$470200 \mathrm{ml}$ of $5 \mathrm{X}$ minimal salts buffer, $1 \mathrm{ml}$ of $1 \mathrm{M}$ magnesium sulphate, $0.1 \mathrm{ml}$ of calcium 471 sulphate, $25 \mathrm{ml}$ of $200 \mathrm{~g} / 1$ solution of casamino acids (Bacto TM from BD, Sparks,MD) with 
$0.5 \%$ of agar. $5 \mathrm{X}$ stock minimal salts buffer was prepared with $12 \mathrm{~g}$ of $\mathrm{Na}_{2} \mathrm{HPO} 4$ (anhydrous), $15 \mathrm{~g}$ of $\mathrm{KH}_{2} \mathrm{PO} 4$ (anhydrous) and $2.5 \mathrm{~g}$ of $\mathrm{NaCl}$ into 11 water.

474 Succinate and glycerol growth curve assays. The clinical isolates were inoculated in $3 \mathrm{~mL}$

475 of lysogen broth (LB) and incubated at $37^{\circ} \mathrm{C}$ overnight with shaking at $250 \mathrm{rpm} .500 \mu \mathrm{L}$ of 476 cell culture was pelleted and washed 3 times with PBS. $0.0025 \mathrm{OD}_{600}$ units were inoculated 477 into $3.0 \mathrm{gC} / 1$ glycerol or succinate minimal medium in BD Falcon (BD Biosciences, San Jose, 478 CA) 96 well flat-bottom plates, with $150 \mu \mathrm{L}$ of suspension per well. The plate was incubated 479 during 48 hours at $37^{\circ} \mathrm{C}$ in a Tecan Infinite M1000 or Tecan Infinite M1000 Pro plate reader 480 (Männedorf, Switzerland), with an orbital shaking of $4 \mathrm{~mm}$ of amplitude. OD $_{600}$ was 481 measured in 10 minutes intervals.

482 Swarming assay Swarming assays were performed as described previously (Xavier et al, 483 2011; van Ditmarsch et al, 2013). The clinical isolates were inoculated in $3 \mathrm{~mL}$ of LB and incubated at $37^{\circ} \mathrm{C}$ overnight, with shaking. $500 \mu \mathrm{L}$ of the culture was pelleted and washed twice with PBS. $2 \mu \mathrm{L}$ of this suspension was spotted on the surface of swarming agar without penetrating the agar with the pipette tip. Swarming medium contains $0.5 \%$ agar (Bacto) and is supplemented with $5 \mathrm{~g} / \mathrm{L}$ casamino acids, $1 \mathrm{mM} \mathrm{MgSO} 4,0.1 \mathrm{mM} \mathrm{CaCl} 2$ and $1 \mathrm{X}$ salt buffer $\left(12 \mathrm{~g} / \mathrm{L} \mathrm{Na}_{2} \mathrm{HPO}_{4}\right.$ (Fisher Scientific), $15 \mathrm{~g} / \mathrm{L} \mathrm{KH}_{2} \mathrm{PO} 4$ (Fisher Scientific) and $2.5 \mathrm{~g} / \mathrm{L} \mathrm{NaCl}$, pH6.7). The plates were incubated at $37^{\circ} \mathrm{C} \sim 24$ hours. Two replicates were done per strain. In each batch of swarming assays PA14 was used as control.

491 Rhamnolipid production quantification. The production of rhamnolipids was assessed by 492 drop-collapse assay (Jain \& Collins; Chen et al, 2007). We placed $50 \mu \mathrm{L}$ of the culture's 493 supernatant on a polystyrene surface (the lid of a 96 well plate). The presence of 494 rhamnolipids decreases the surface tension of the liquid, making the drop collapse. We 495 considered a strain rhamnolipid non-producer if the drop does not spread, a mild-producer if 
spreads, but not enough to pass over the lid's circle that correspond to the wells, and producer

497 if spreads over it.

498 Image analysis and quantification of swarming phenotype. Images of the swarming plates

499 were obtained with a Chemidoc gel doc imager (Bio Rad). The extraction of the

500 morphological features from the images of the swarming plates were analyzed using Matlab

501 bwmorph function. The features extracted from the images were perimeter of the colony,

502 maximum length (the longitude of the rectangle that fits the colony), area percentage of the

503 plate occupied by the colony, circularity (measured as $4^{*} \pi / \mathrm{P}^{2}$, where $\mathrm{P}$ is the perimeter of the

504 colony), longitude of the skeleton (skeletonization, consisting in all the points that are at

505 equal distance to their nearby borders) and eccentricity (the eccentricity of the fitted ellipse).

506 The feature values of each strain were normalized by the corresponding values of the PA14

507 strain in the same experiment. The swarming score $\left(S_{i}\right)$ for each clinical isolate $i$ is defined as

508 a linear combination of the maximum length $\left(L_{i}\right)$ and circularity $\left(C_{i}\right)$ of its swarming colony

509 (the two most discriminative features that separate strong swarmers from weak swarmers; see

510 Supplementary Fig. 1)

$$
S_{i}=0.60\left(L_{i}-\tilde{L}\right)-0.80\left(C_{i}-\tilde{C}\right)
$$

511 where $\tilde{L}$ and $\tilde{C}$ are the mean values of $L_{i}$ and $C_{i}$ across all clinical isolates respectively, and

512 the linear coefficients maximize the variance of the swarming score. To binarize the 513 swarming phenotype, a strain is called swarmer if its swarming score is higher than the

514 median swarming score across all strains (otherwise it is a non-swarmer).

515 Cross-strain reciprocal BLAST. To identify orthologous gene across all $P$. aeruginosa

516 strains, systematic cross-strain reciprocal BLAST was performed. Protein sequences of each

517 strain and the type strain PA14 were mutually queried against and the BLAST hits (genes) 
518 with $<80 \%$ coverage were removed. Two genes are orthologous if they were mutually the

519 best hit to each other and genes (from other strains) that are orthologous to the same PA14

520 gene were considered orthologous to each other.

521 Phylogenetic analysis. The phylogenetic tree of clinical P. aeruginosa strains (Fig. 1A) was

522 constructed from core genomes as previously described (Yan et al, 2017). Moran's I test was

523 carried out using the ape package in R (Paradis \& Schliep, 2019). The ancestral state of

524 swarming and rhamnolipid production was reconstructed using corHMM package (Beaulieu et al, 2013).

Growth curve analysis. We first normalized the growth curves in different experiments (plates) by matching their corresponding controls (PA14). The plate-specific coefficients were obtained by solving a linear regression model ' $\log (\mathrm{OD}$ of PA14) $\sim$ Plate + Time' (the Wilkinson notation) using Matlab function fitlm, where Plate is plate ids and Time is time points (both are categorical variables). Then the growth curves in each plate were normalized by multiplying the adjustment coefficient of the plate. These normalized growth curves were then smoothed by Savitzky-Golay filter (sgolayfilt function in Matlab). The growth phases (phase I, II, III) were determined by the following rules: (phase I): first-order derivative $>0$ and second-order derivative $>0$; (phase II) first-order derivative $>0$ and second-order derivative $<0$; (phase III) first-order derivative $<0$. The mean and maximum specific growth rate for each growth phase were estimated from the best-fit model (highest R2) among three

538 Logistic model $y=y_{0}+\left(A-y_{0}\right)\left(1+e^{\frac{4 \mu}{A}(\lambda-t)+2}\right)^{-1} ;$ (3) Zwietering-Gompertz model $539 y=y_{0}+\left(A-y_{0}\right) e^{-e^{\frac{\mu e}{A}(\lambda-t)+1}}$, where $y_{0}, y_{1}, \mu, \lambda, A$ are the parameters to fit. Both non540 negative factorization and random forest regression were performed in python, by 541 sklearn.decomposition.NMF and sklean.ensemble.RandomForestClassifier respectively. 
542 Metabolite extraction. All $P$. aeruginosa strains were grown until $\mathrm{OD}_{600}=0.2$ (end of

543 exponential phase of growth) in glycerol minimal media. Bacteria was then loaded into 0.25

$544 \mu \mathrm{m}$ nylon membranes (Millipore) using vacuum, transferred to pre-warmed hard agar plates

545 with the same medium composition and incubated at $37^{\circ} \mathrm{C}$ during $2.5 \mathrm{~h}$. The filters were then

546 passed to $35 \mathrm{~mm}$ polystyrene dishes (Falcon) with $1 \mathrm{~mL}$ of 2:2:1 methanol:acetonitrile: $\mathrm{H}_{2} \mathrm{O}$

547 quenching buffer and incubated there during 15 minutes on dry ice. Cells were removed by

548 scraping and the lysate containing quenching buffer was transferred to $1.5 \mathrm{~mL}$ tubes and

549 centrifuged at $16000 \mathrm{rpm}$ for 10 minutes at $4^{\circ} \mathrm{C}$. Supernatant transferred to fresh tubes and

550 stored at $-80^{\circ} \mathrm{C}$.

551 Metabolomic data preprocessing. The extracts were profiled using liquid-chromatography

552 coupled to mass spectrometry (LC-MS), identifying a total of 92 compounds (Supplementary

553 Fig. 5). Some compounds contained missing values. These missing values in metabolite

554 abundance can be (1) truly missing; (2) present in a sample but its level is below detection

555 limit; (3) present in a sample at a level above the detection limit but missing due to failure of

556 algorithms in data processing. Here we assume that a metabolite with missing values in all

557 three replicates is truly missing in the sample and removed from our analysis (Supplementary

558 Fig. 5). However, if the missing values were only found in one or two replicates, the missing

559 values were imputed by the average of the non-missing values. After that imputation all

560 compounds with missing values were removed (Supplementary Fig. 5).

561 The peak areas were normalized using Cross-Contribution Compensating Multiple Standard

562 Normalization (CCMN) (Redestig et al, 2009) with NormalizeMets R package (De Livera et 563 al, 2018). This method relies on the use of multiple internal standards. Since LC-MS lacks

564 such internal standards, we used instead a set of metabolites assumed to be constant across all 565 the strains. They were selected with a Kuskal-Wallis test, adjusting the $p$-value with 
566 Benjamini-Hochberg method. The ones with a $p$-value above 0.05 were considered constant

567 (pyruvate, methylglyoxal, (S)-2-Acetolactate, Tyramine, D-Glucose, (S)-Lactate, N-acetyl-L-

568 glutamate 5-semialdehyde, 4-Aminobutyraldehyde and Glycine), therefore after the

569 normalization step they were removed (indicated in red, Fig. 3A).

570 Hierarchical clustering of metabolomic data. The hierarchical clustering of the normalized

571 metabolomic data (Fig. 3B) was performed using gplots R package (Warnes et al. 2009), with

572 Euclidean distance and Ward's aggregation method. The clustering was performed with all metabolites; however, 16 metabolites (indicated in red, Fig. 3A) with unknown compound identity were removed from Fig. 3B and downstream analyses. These unknown compounds are not artifacts arising from LC-MS instruments since their peaks were detected in all strains. Fumarate and guanosine, which were only putative initially, were bioinformatically confirmed as all other compounds with the same molecular weight are produced by enzymatic reactions missing in our clinical isolates.

Metabolic pathway enrichment. The differential metabolites between rhamnolipid

580 producers and non-producers was determined by a Mann-Whitney test, with $p$-values

581 adjusted with Benjamini-Hochberg method and a significance level of 0.05 . These

582 compounds were fed to FELLA algorithm (Picart-Armada et al, 2018, 2017). FELLA

583 retrieves a graph describing the relationships among pathway, module, enzyme, reaction and compounds of Pseudomonas aeruginosa strain UCBPP-PA14 from the KEGG database. The graph was then used as an input network of differential compounds for its diffusion

586 algorithms (Vandin et al 2011). The output of FELLA consists of all subnetworks of the 587 entries predicted to have a high connectivity with the differential compounds. We filtered the subnetwork entries to keep only metabolic pathways. 
589 OPLS-DA model. OPLS-DA model of metabolomics data was built using ropls R package

590 (Thévenot et al, 2015), fixing the number of orthogonal components to $3 . \mathrm{R}^{2}$ and $\mathrm{Q}^{2}$, key

591 parameters for assessing the validity of the model, were assessed with 7-fold cross validation.

592 The significance of the model was determined by permutation test $(\mathrm{n}=2000)$. The $p$-value

593 corresponds to the proportion of $\mathrm{Q}^{2}$ perm above $\mathrm{Q}^{2}$. With a $p$-value below 0.05 we considered

594 the model significant. The loadings of the predictive component of the model were extracted

595 to determine how each metabolite contributes to the separation according to the phenotype.

596 Genome-scale modeling. Custom Python codes were developed with the COBRApy

597 package (Ebrahim et al, 2013) to carry out all metabolic flux modeling and simulations in the

598 paper. Since iJN1411 model was developed for Pseudomonas putida, we removed genes and

599 associated reactions that are missing in all our strains but present in the iJN1411 model. The

600 futile cycles involving NADH, NADPH, and GSH were also removed. The modified iJN1411

601 model was further expanded by adding rhamnolipid biosynthesis pathway involving 9 new

602 metabolites and 12 new reactions. The metabolites, reactions and genes of the final model are

603 given in JSON format (Supplementary File 4).

604 The boundary fluxes of the model were set to mimic the composition of the glycerol 605 minimum medium. For $\mathrm{C}: \mathrm{N}=10$, the lower bounds of glycerol and ammonium fluxes were set 606 to -10 and -3 respectively. For $\mathrm{C}: \mathrm{N}=3$, the lower bounds were set to -3 and -3 respectively.

607 The flux unit is $\mathrm{mmol} / \mathrm{gDW} / \mathrm{h}$ throughout the paper. To constrain the total producing flux of 608 NADH (the same for NADPH and GSH) at a certain value $C$, we first defined a binary 609 variable $X_{k}$ for each NADH-involving reaction $k$ to indicate whether NADH is produced by 610 this reaction. Given the stoichiometric coefficient of NADH in this reaction $\left(s_{k}\right)$ and its flux

611 value $\left(f_{k}\right)$, the mathematical constraints for $X_{k}$ was set by $X_{k}=1$ for $s_{k} f_{k}>0$ and $X_{k}=0$ 612 otherwise. Therefore, the constraint that equalizes the total NADH producing flux and a 
613 constant $C$ is simply $\sum_{k} s_{k} X_{k} f_{k}=C$. However, both $X_{k}$ and $f_{k}$ are variables and such

614 quadratic constraint has not yet been supported by COBRApy. We overcame this difficulty

615 by defining $A_{k}=X_{k} f_{k}$ and linearized the product with the following two inequalities:

$616 X_{k} l_{b} \leq A_{k} \leq X_{k} u_{b}$ and $f_{k}-\left(1-X_{k}\right) u_{b} \leq A_{k} \leq f_{k}-\left(1-X_{k}\right) l_{b}$, where $l_{b}$ and $u_{b}$ are the

617 lower and upper bounds of $f_{k}$. The two constraints ensure that $A_{k}=0$ when $X_{k}=0$ and

$618 A_{k}=f_{k}$ when $X_{k}=1$. The minimum/maximum flux values of byproduct secretion were

619 simulated by flux variability analysis at maximum growth rate.

620 Detection of hydrogen peroxide $\left(\mathbf{H}_{2} \mathbf{O}_{2}\right)$. The $\mathrm{H}_{2} \mathrm{O}_{2}$ level in the extracellular medium was quantified with Amplex ${ }^{\circledR}$ Red Hydrogen Peroxide/Peroxidase Assay Kit (Invitrogen, Carlsbad, USA, Catalog no. A22188). $500 \mu \mathrm{L}$ of cell suspension were spinned down after overnight growth in LB medium, washed twice in PBS and normalized to OD 1. Each 624 reaction was done in a final volume of $100 \mu \mathrm{L}$, with a final concentration of $50 \mu \mathrm{M}$ of 625 Amplex ${ }^{\circledR}$ Red reagent, $0.1 \mathrm{U} / \mathrm{mL}$ of HRP (Horseradish Peroxidase) and 0.2 cell OD, in glycerol synthetic medium, in BD Falcon (BD Biosciences, San Jose, CA) 96 well flatbottom plates. The first column of the plate corresponded to the reaction without cells (the volume was substituted by PBS), and the last column instead of cells contained $\mathrm{H}_{2} \mathrm{O}_{2}$ (final concentration $10 \mu \mathrm{M}) . \mathrm{OD}_{600}$ and fluorescence $530 / 590 \mathrm{~nm}$ was measured in 10 minutes 630 intervals $\left(48 \mathrm{~h} 37^{\circ} \mathrm{C}\right)$.

631 Hydrogen peroxide $\left(\mathbf{H}_{2} \mathbf{O}_{2}\right)$ degradation. $\mathrm{H}_{2} \mathrm{O}_{2}$ was removed from environment by cells 632 which degrade $\mathrm{H}_{2} \mathrm{O}_{2}$ intracellularly. The cumulative $\mathrm{H}_{2} \mathrm{O}_{2}$ removal curve was calculated by 633 subtracting the values of emission of the wells containing each strain from the values of 634 emission of the wells without cells or $\mathrm{H}_{2} \mathrm{O}_{2}$. Then they were normalized to the relative scale 635 by dividing their values by the corresponding values (at the same time points) of the control strain (PA14) in the same experiment (plate). These normalized curves were then smoothed 
637 by the Savitzky-Golay filter (sgolayfilt function in Matlab). The $\mathrm{H}_{2} \mathrm{O}_{2}$ removal rate per cell

638 was calculated by dividing the first-order derivative of the cumulative removal curves by OD

639 values. To estimate the effect of rhamnolipid production on per-cell $\mathrm{H}_{2} \mathrm{O}_{2}$ removal rate, we

640 developed a linear mixed-effect model that extends the simple linear regression model by

641 considering both fixed and random effects. The model was specified by the Wilkinson

642 notation 'RemovalRate $\sim$ Time + RHL + (1|CurveID)', where RemovalRate is per-cell $\mathrm{H}_{2} \mathrm{O}_{2}$

643 removal rate, Time is time point (categorical variable), RHL is categorical rhamnolipid

644 production (non-producer, mild-producer, and strong-producer), and CurveID is the unique

645 ID for each strain and replicate. The expression '(1|CurveID)' indicates that CurveID was

646 modeled as random effects. The model was solved by fitlme in Matlab.

\section{Data availability}

649 All experimental and simulation data that support the conclusions of this study are available

650 from the main text and Supplementary Information. The source codes for generating the

651 figures of this study are available from

652 https://github.com/liaochen1988/Source_code_for_Pseudomonas_Metabolomics_Paper.

\section{Acknowledgements}

654 This work was supported by NIH grants U01 AI124275 and R01 AI137269-01 to J.B.X.

655 F.R.P. and G.S. were partially supported by UIDB/04046/2020 and UIDP/04046/2020 Centre

656 grants from FCT, Portugal (to BioISI) and by the LungCARD project (Grant Agreement $\mathrm{n}^{\mathrm{o}}$ :

657 734790, H2020-MSCA-RISE-2016). G.S. is recipient of a fellowship from BioSys PhD 658 programme PD65-2012 (Ref SFRH/BD/142899/2018) from FCT (Portugal). The funders had 
no role in study design, data collection and analysis, decision to publish, or preparation of the

660 manuscript.

661

\section{References}

663 Abdel-Mawgoud AM, Lépine F \& Déziel E (2010) Rhamnolipids: diversity of structures, microbial origins and roles. Appl. Microbiol. Biotechnol. 86: 1323-1336

665

Altindis E, Dong T, Catalano C \& Mekalanos J (2015) Secretome analysis of Vibrio cholerae

666 type VI secretion system reveals a new effector-immunity pair. MBio 6: e00075

Ayala-Castro C, Saini A \& Outten FW (2008) Fe-S cluster assembly pathways in bacteria. Microbiol. Mol. Biol. Rev. 72: 110-25, table of contents

Basan M, Hui S, Okano H, Zhang Z, Shen Y, Williamson JR \& Hwa T (2015) Overflow metabolism in Escherichia coli results from efficient proteome allocation. Nature 528: $99-104$

Beaulieu JM, O'Meara BC \& Donoghue MJ (2013) Identifying hidden rate changes in the evolution of a binary morphological character: the evolution of plant habit in campanulid angiosperms. Syst. Biol. 62: 725-737

Biro PA \& Stamps JA (2010) Do consistent individual differences in metabolic rate promote consistent individual differences in behavior? Trends Ecol. Evol. (Amst.) 25: 653-659

677 Boyle KE, Monaco H, van Ditmarsch D, Deforet M \& Xavier JB (2015) Integration of metabolic and quorum sensing signals governing the decision to cooperate in a bacterial social trait. PLoS Comput. Biol. 11: e1004279

Boyle KE, Monaco HT, Deforet M, Yan J, Wang Z, Rhee K \& Xavier JB (2017) Metabolism and the evolution of social behavior. Mol. Biol. Evol. 34: 2367-2379

682 Cai Y, Chandrangsu P, Gaballa A \& Helmann JD (2017) Lack of formylated methionyl- 

$185-196$

Caiazza NC, Shanks RMQ \& O’Toole GA (2005) Rhamnolipids modulate swarming motility patterns of Pseudomonas aeruginosa. J. Bacteriol. 187: 7351-7361

Chen CY, Baker SC \& Darton RC (2007) The application of a high throughput analysis method for the screening of potential biosurfactants from natural sources. $J$. Microbiol. Methods

Chong H \& Li Q (2017) Microbial production of rhamnolipids: opportunities, challenges and strategies. Microb. Cell Fact. 16: 137

692 Cloarec O, Dumas M-E, Craig A, Barton RH, Trygg J, Hudson J, Blancher C, Gauguier D, an exploratory approach for latent biomarker identification from metabolic $1 \mathrm{H}$ NMR data sets. Anal. Chem. 77: 1282-1289

De Deken R (1966) The Crabtree Effect: A Regulatory System in Yeast. J. Gen. Microbiol.

De Livera AM, Olshansky G, Simpson JA \& Creek DJ (2018) NormalizeMets: assessing, selecting and implementing statistical methods for normalizing metabolomics data. Metabolomics 14: 54

Deforet M, van Ditmarsch D, Carmona-Fontaine C \& Xavier JB (2014) Hyperswarming adaptations in a bacterium improve collective motility without enhancing single cell motility. Soft Matter 10: 2405-2413

Déziel E, Lépine F, Milot S \& Villemur R (2003) rhlA is required for the production of a novel biosurfactant promoting swarming motility in Pseudomonas aeruginosa: 3-(3hydroxyalkanoyloxy)alkanoic acids (HAAs), the precursors of rhamnolipids. Microbiology (Reading, Engl.) 149: 2005-2013 

Xavier JB (2013) Convergent evolution of hyperswarming leads to impaired biofilm formation in pathogenic bacteria. Cell Rep. 4: 697-708

van Ditmarsch D \& Xavier JB (2011) High-resolution time series of Pseudomonas aeruginosa gene expression and rhamnolipid secretion through growth curve synchronization. BMC Microbiol. 11: 140

Ebrahim A, Lerman JA, Palsson BO \& Hyduke DR (2013) COBRApy: COnstraints-Based Reconstruction and Analysis for Python. BMC Syst. Biol. 7: 74

Ezraty B, Gennaris A, Barras F \& Collet J-F (2017) Oxidative stress, protein damage and repair in bacteria. Nat. Rev. Microbiol. 15: 385-396

Farmer IS \& Jones CW (1976) The energetics of Escherichia coli during aerobic growth in continuous culture. Eur. J. Biochem. 67: 115-122

Griffin AS, West SA \& Buckling A (2004) Cooperation and competition in pathogenic bacteria. Nature 430: 1024-1027

Hederstedt L \& Rutberg L (1981) Succinate dehydrogenase--a comparative review. Microbiol Rev 45: 542-555

Inoue T, Shingaki R, Hirose S, Waki K, Mori H \& Fukui K (2007) Genome-wide screening $950-957$

Jain DK \& Collins DL A drop-collapsing test for screening surfactant-producing microorganisms. Thompson

Kamatkar NG \& Shrout JD (2011) Surface hardness impairment of quorum sensing and swarming for Pseudomonas aeruginosa. PLoS One 6: e20888 
733 Kim W \& Surette MG (2004) Metabolic differentiation in actively swarming Salmonella. Mol. Microbiol. 54: 702-714

Klevens RM, Edwards JR, Richards CL, Horan TC, Gaynes RP, Pollock DA \& Cardo DM (2007) Estimating health care-associated infections and deaths in U.S. hospitals, 2002. Public Health Rep. 122: 160-166

Köhler T, Curty LK, Barja F, van Delden C \& Pechère JC (2000) Swarming of Pseudomonas aeruginosa is dependent on cell-to-cell signaling and requires flagella and pili. $J$. Bacteriol. 182: 5990-5996

Kollaran AM, Joge S, Kotian HS, Badal D, Prakash D, Mishra A, Varma M \& Singh V (2019) Context-Specific Requirement of Forty-Four Two-Component Loci in Pseudomonas aeruginosa Swarming. iScience 13: 305-317

Lee DD \& Seung HS (1999) Learning the parts of objects by non-negative matrix factorization. Nature 401: 788-791

Lequette Y, Lee J-H, Ledgham F, Lazdunski A \& Greenberg EP (2006) A distinct QscR regulon in the Pseudomonas aeruginosa quorum-sensing circuit. J. Bacteriol. 188: $3365-3370$

Luo Y, Zhao K, Baker AE, Kuchma SL, Coggan KA, Wolfgang MC, Wong GC \& O’Toole GA (2015) A hierarchical cascade of second messengers regulates Pseudomonas aeruginosa surface behaviors. MBio 6:

Mattingly AE, Kamatkar NG, Borlee BR \& Shrout JD (2018) Multiple Environmental Factors Influence the Importance of the Phosphodiesterase DipA upon Pseudomonas aeruginosa Swarming. Appl. Environ. Microbiol. 84: 
Mellbye B \& Schuster M (2014) Physiological framework for the regulation of quorum sensing-dependent public goods in Pseudomonas aeruginosa. J. Bacteriol. 196: $1155-1164$

Mishra S \& Imlay J (2012) Why do bacteria use so many enzymes to scavenge hydrogen peroxide? Arch. Biochem. Biophys. 525: 145-160

Mohamed FA, Shaker GH \& Askoura MM (2020) Oxidative Stress Influences Pseudomonas aeruginosa Susceptibility to Antibiotics and Reduces Its Pathogenesis in Host. Curr Microbiol 77: 479-490

Monaco H, Sereno T, Liu K, Reagor C, Deforet M \& Xavier JB (2020) Spatial-temporal dynamics of a microbial cooperative behavior robust to cheating. BioRxiv

Mulvey MR, Sorby PA, Triggs-Raine BL \& Loewen PC (1988) Cloning and physical characterization of $k a t E$ and $k a t F$ required for catalase HPII expression in Escherichia coli. Gene 73: 337-345

Nogales J, Gudmundsson S, Duque E, Ramos JL \& Palsson BO (2017) Expanding The Computable Reactome In Pseudomonas putida Reveals Metabolic Cycles Providing Robustness. BioRxiv

Nogales J, Mueller J, Gudmundsson S, Canalejo FJ, Duque E, Monk J, Feist AM, Ramos JL, Niu W \& Palsson BO (2020) High-quality genome-scale metabolic modelling of Pseudomonas putida highlights its broad metabolic capabilities. Environ. Microbiol. 22: $255-269$

Overhage J, Lewenza S, Marr AK \& Hancock REW (2007) Identification of genes involved in swarming motility using a Pseudomonas aeruginosa PAO1 mini-Tn5-lux mutant library. J. Bacteriol. 189: 2164-2169

Paradis E \& Schliep K (2019) ape 5.0: an environment for modern phylogenetics and evolutionary analyses in R. Bioinformatics 35: 526-528 
Perry AC, Ni Bhriain N, Brown NL \& Rouch DA (1991) Molecular characterization of the gor gene encoding glutathione reductase from Pseudomonas aeruginosa: determinants of substrate specificity among pyridine nucleotide-disulphide oxidoreductases. Mol. Microbiol. 5: 163-171

Pessi G \& Haas D (2000) Transcriptional control of the hydrogen cyanide biosynthetic genes $h c n A B C$ by the anaerobic regulator ANR and the quorum-sensing regulators Las R and RhlR in Pseudomonas aeruginosa. J. Bacteriol. 182: 6940-6949

Piatkov KI, Vu TTM, Hwang C-S \& Varshavsky A (2015) Formyl-methionine as a degradation signal at the N-termini of bacterial proteins. Microb. Cell 2: 376-393

Picart-Armada S, Fernández-Albert F, Vinaixa M, Rodríguez MA, Aivio S, Stracker TH, Yanes O \& Perera-Lluna A (2017) Null diffusion-based enrichment for metabolomics data. PLoS One 12: e0189012

Picart-Armada S, Fernández-Albert F, Vinaixa M, Yanes O \& Perera-Lluna A (2018) FELLA: an R package to enrich metabolomics data. BMC Bioinformatics 19: 538 Puertollano MA, Puertollano E, de Cienfuegos GÁ \& de Pablo MA (2011) Dietary antioxidants: immunity and host defense. Curr. Top. Med. Chem. 11: 1752-1766

Py B \& Barras F (2010) Building Fe-S proteins: bacterial strategies. Nat. Rev. Microbiol. 8:

$$
436-446
$$

Redestig H, Fukushima A, Stenlund H, Moritz T, Arita M, Saito K \& Kusano M (2009) Compensation for systematic cross-contribution improves normalization of mass spectrometry based metabolomics data. Anal. Chem. 81: 7974-7980

Schmidt R, Ulanova D, Wick LY, Bode HB \& Garbeva P (2019) Microbe-driven chemical ecology: past, present and future. ISME J. 13: 2656-2663

Smith E \& Morowitz HJ (2004) Universality in intermediary metabolism. Proc. Natl. Acad. Sci. USA 101: 13168-13173 
Stickland HG, Davenport PW, Lilley KS, Griffin JL \& Welch M (2010) Mutation of $n f x B$

Szenk M, Dill KA \& de Graff AMR (2017) Why Do Fast-Growing Bacteria Enter Overflow Metabolism? Testing the Membrane Real Estate Hypothesis. Cell Syst. 5: 95-104

813 Thévenot EA, Roux A, Xu Y, Ezan E \& Junot C (2015) Analysis of the Human Adult Implementing a Comprehensive Workflow for Univariate and OPLS Statistical Analyses. J. Proteome Res. 14: 3322-3335

817 Tremblay J \& Déziel E (2010) Gene expression in Pseudomonas aeruginosa swarming motility. BMC Genomics 11: 587

819 Vander Heiden MG, Cantley LC \& Thompson CB (2009) Understanding the Warburg effect: the metabolic requirements of cell proliferation. Science 324: 1029-1033 microbial social trait. Mol. Syst. Biol. 9: 684

823 Vemuri GN, Altman E, Sangurdekar DP, Khodursky AB \& Eiteman MA (2006) Overflow metabolism in Escherichia coli during steady-state growth: transcriptional regulation and effect of the redox ratio. Appl. Environ. Microbiol. 72: 3653-3661 oxidation reduces overflow metabolism in Saccharomyces cerevisiae. Proc. Natl. Acad. Sci. USA 104: 2402-2407

Vinckx T, Wei Q, Matthijs S \& Cornelis P (2010) The Pseudomonas aeruginosa oxidative stress regulator OxyR influences production of pyocyanin and rhamnolipids: protective role of pyocyanin. Microbiology (Reading, Engl.) 156: 678-686

832 Westbrock-Wadman S, Sherman DR, Hickey MJ, Coulter SN, Zhu YQ, Warrener P, Nguyen 
836 Xavier JB (2011) Social interaction in synthetic and natural microbial communities. Mol. Syst. Biol. 7: 483

Xavier JB, Kim W \& Foster KR (2011) A molecular mechanism that stabilizes cooperative secretions in Pseudomonas aeruginosa. Mol. Microbiol. 79: 166-179

840 Xiao W \& Loscalzo J (2019) Metabolic responses to reductive stress. Antioxid. Redox Signal.

841 Yan J, Deforet M, Boyle KE, Rahman R, Liang R, Okegbe C, Dietrich LEP, Qiu W \& Xavier JB (2017) Bow-tie signaling in c-di-GMP: Machine learning in a simple biochemical network. PLoS Comput. Biol. 13: e1005677

844 Yan J, Estanbouli H, Liao C, Kim W, Monk JM, Rahman R, Kamboj M, Palsson BO, Qiu W \& Xavier JB (2019) Systems-level analysis of NalD mutation, a recurrent driver of rapid drug resistance in acute Pseudomonas aeruginosa infection. PLoS Comput. Biol. 15: e1007562

Yeung ATY, Bains M \& Hancock REW (2011) The sensor kinase CbrA is a global regulator that modulates metabolism, virulence, and antibiotic resistance in Pseudomonas

Zhao B, Summers FA \& Mason RP (2012) Photooxidation of Amplex Red to resorufin: implications of exposing the Amplex Red assay to light. Free Radic. Biol. Med. 53:

854 Zhu K, Chen S, Sysoeva TA \& You L (2019) Universal antibiotic tolerance arising from antibiotic-triggered accumulation of pyocyanin in Pseudomonas aeruginosa. PLoS Biol. 17: e3000573

857 Zhu K \& Rock CO (2008) RhlA converts beta-hydroxyacyl-acyl carrier protein intermediates 
863 Table 1 Genes that are missing in non-rhamnolipid-producers but present in all

864 producers

\begin{tabular}{|c|c|}
\hline Non-producers & Missing genes \\
\hline F5677 & $\operatorname{ars} B, \operatorname{ars} C, \operatorname{ars} H, \operatorname{ars} R, \operatorname{chiC}, d a c B, f l i M$, gcd, $q s c R$, gntK, gntR, \\
\hline F63912 & lasR \\
\hline H27930 & arn $A$, pelA, pcaB, pcaD, pfeA, icmF3, rrsmE, pcd, pirR, pctA, \\
\hline M1608 & $\begin{array}{l}\text { tauD, opd } G, p d x A, \text { hcn } C, \text { hcnB, hcn } A, g \lg A, y b h O, g l g B, \text { katE, } \\
\text { glgP, ligD, ada, opdO, kynB, fusA2, cynR, cynT, cynS, cmrA, gor, } \\
\text { galU, amrB, gnyD, gnyB, gnyH, gny } A, \text { gnyL, hmgA, fahA, maiA, }\end{array}$ \\
\hline M55212 & crc, rph \\
\hline S86968 & $k d s B, c y s P, h c n C, h c n B, h c n A, p d x A$, opdG, nfxB, cupB3 \\
\hline W36662 & rhlR, rhlI, cupC1, pilC, pilS \\
\hline W60856 & lasR, pirA \\
\hline
\end{tabular}

865 


\section{Figures}

867
A

B

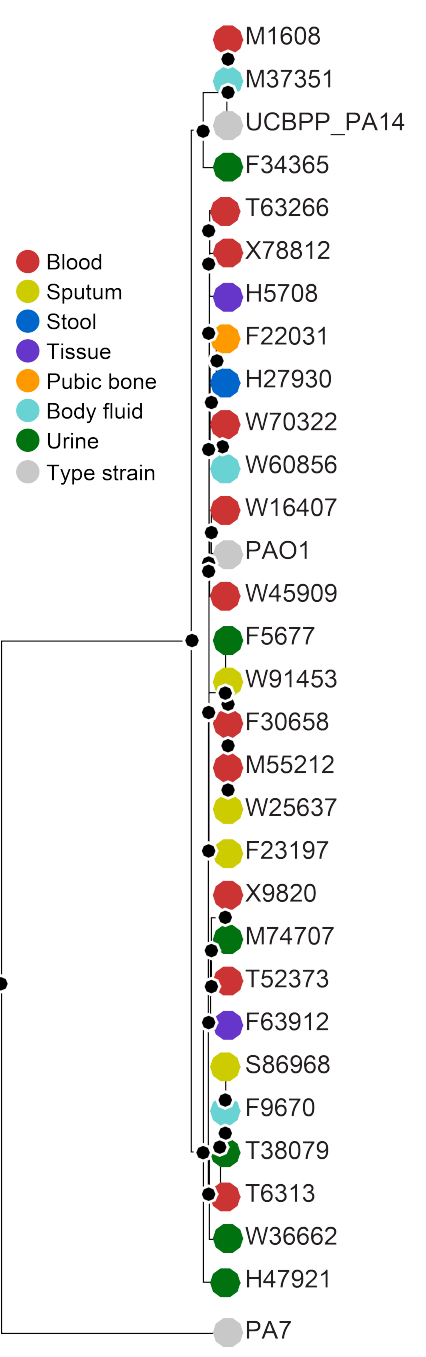

Swarm

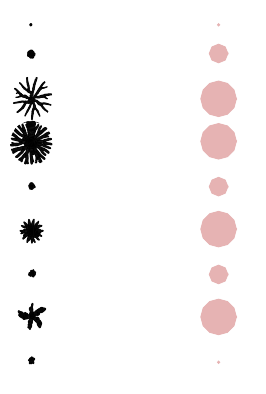

$\bullet$

$\cdot$

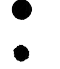

-

.

*

$\cdot$

*

*

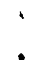

$\cdot$

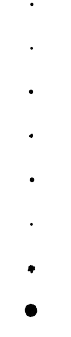

rhamnolipids production
Rhamnolipids C

Growth in glycerol

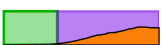

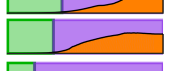

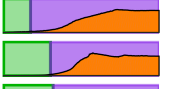

1
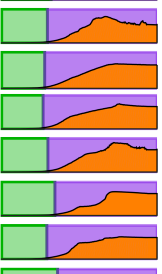

胥
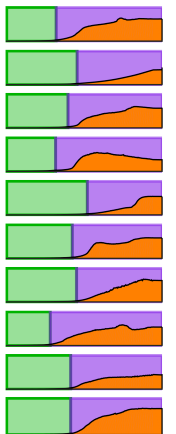

$r$

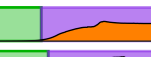

L
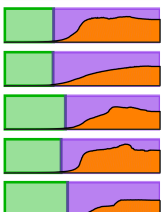

$\begin{array}{ccc}\text { lag } & \text { growth } & \text { filled optical } \\ \text { phase } & \text { phase } & \text { density }\end{array}$

868 Figure 1. Diversity of swarming across the P. aeruginosa phylogeny of core genomes. A. Phylogeny

869 of clinical isolates obtained from patients with cancer at MSKCC (Yan et al, 2017, 2019) together

870 with reference type strains PAO1, PA14 and PA7. The tissue where each isolate was originally

871 isolated is labeled by circle colors. B. Swarming and rhamnolipid production phenotypes. The

872 swarming ability of these strains is represented by the actual swarming images on the agar plates (left

873 column). The ability of producing rhamnolipids of these strains is semi-quantitatively indicated by

874 circle sizes (right column). Both phenotypes do not correlate with phylogeny. All swarmers can 
bioRxiv preprint doi: https://doi.org/10.1101/2020.09.02.280495; this version posted September 3, 2020. The copyright holder for this preprint (which was not certified by peer review) is the author/funder, who has granted bioRxiv a license to display the preprint in perpetuity. It is made available under aCC-BY-ND 4.0 International license.

876 minimal medium using glycerol as the sole carbon source. Strain M6075 was excluded from

877 phylogenetic analysis due to the previous failure in sequencing and assembling its genome.

A

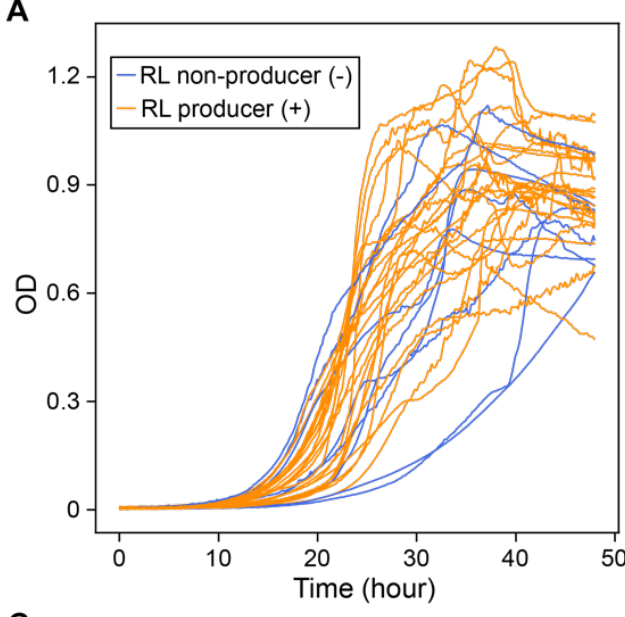

C

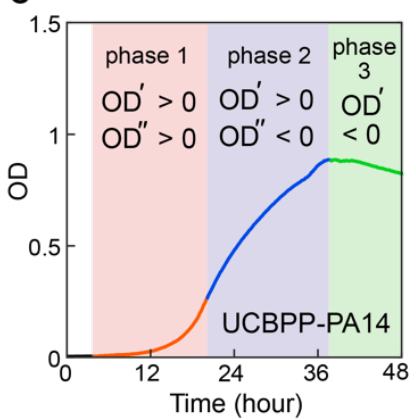

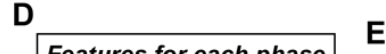

$E$

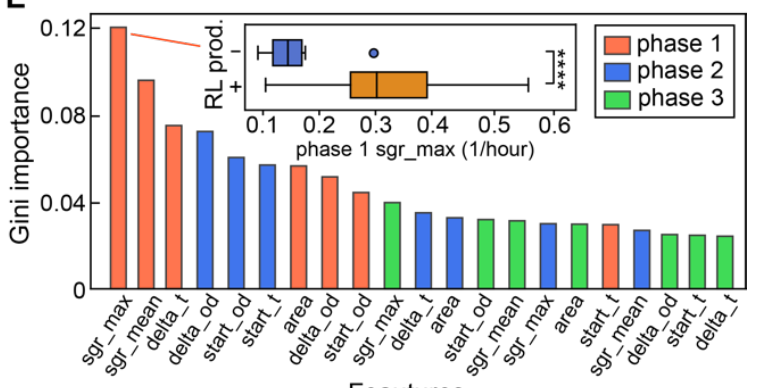

Features for each phase

start time (start_t) duration (delta_t) initial od (init_od) area under the curve (area) mean specific growth rate (sgr_mean) max absolute specific growth rate (sgr_max) od change (delta_od)
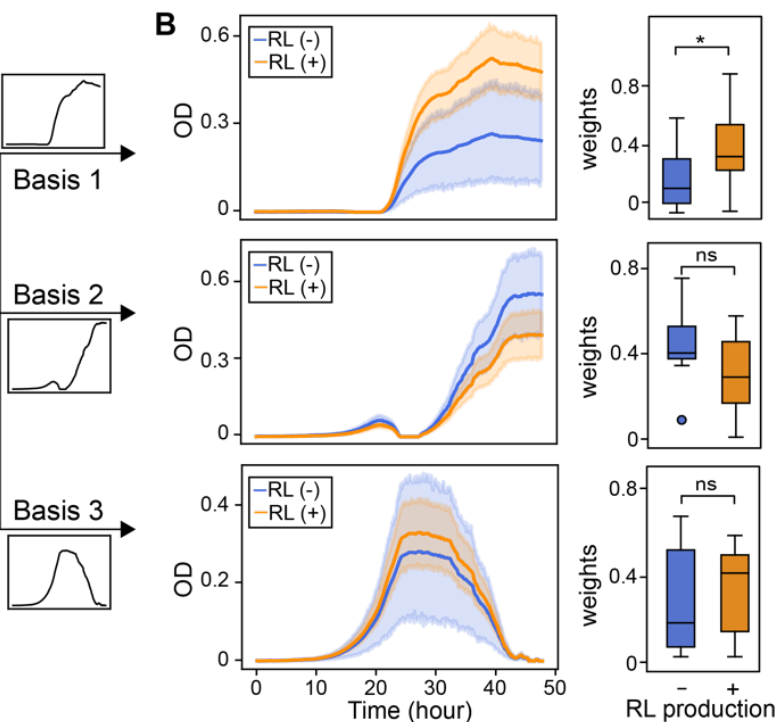

Feautures

879 Figure 2. Growth curve features distinguish rhamnolipid producers from non-producers. A,B.

880 Unsupervised feature selection using non-negative matrix factorization (NNMF), which decomposes

881 growth curves of all Pseudomonas isolates into three additive basis functions (features) such that each

882 growth curve can be approximately represented by the weighted sum of these functions. A. Growth curves from both rhamnolipid producers (orange) and non-producers (blue). B. Decomposed components (basis function multiplied by weights; left panels) and weights (right panels) from NNMF grouped by rhamnolipid (RL) production. The shaded areas represent $95 \%$ bootstrap confidence interval of the mean. C-E. Supervised feature selection using Random Forest classifier. C,D. Feature extraction method. Each growth curve (excluding the initial lag phase) was divided into three phases (C) and each phase was described by 7 quantitative features (D). E. Ranking of feature importance in 
bioRxiv preprint doi: https://doi.org/10.1101/2020.09.02.280495. this version posted September 3, 2020. The copyright holder for this preprint (which was not certified by peer review) is the author/funder, who has granted bioRxiv a license to display the preprint in perpetuity. It is made available under aCC-BY-ND 4.0 International license.

889 classifying rhamnolipid producers. Inset: boxplot of maximum specific growth rate of phase I grouped

890 by rhamnolipid production. Welch's t-test was used in $(\mathbf{B})$ and $(\mathbf{E})$ for significance testing. ****, p-

891 value $\leq 0.0001{ }^{*}$, p-value $\leq 0.05 ; \mathrm{ns}, \mathrm{p}$-value $>0.05$

892
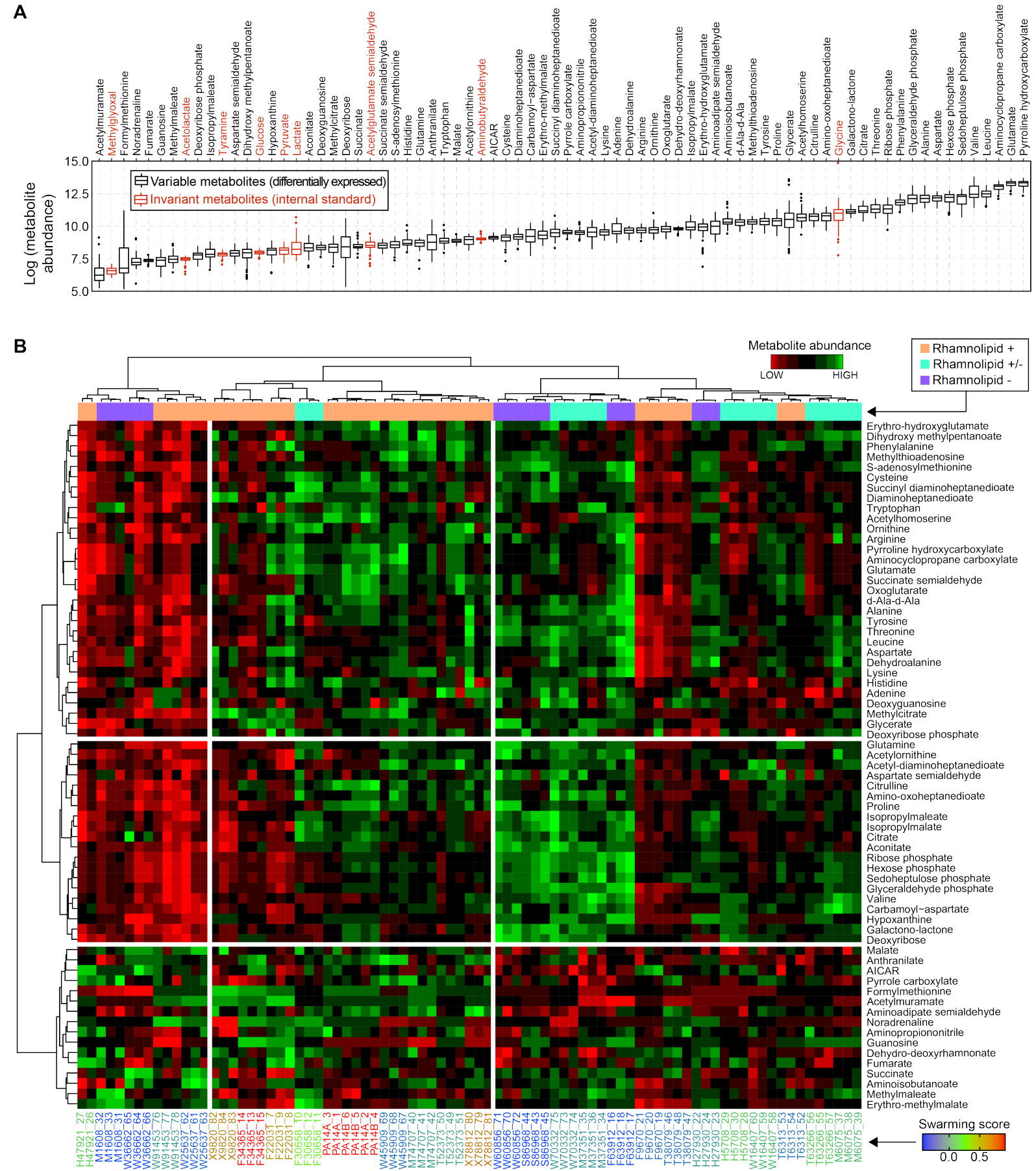
bioRxiv preprint doi: https://doi.org/10.1101/2020.09.02 280495; this version posted September 3, 2020. The copyright holder for this preprint (which was not certified by peer review) is the author/funder, who has granted bioRxiv a license to display the preprint in perpetuity. It is made available under aCC-BY-ND 4.0 International license.

894 Figure 3. Variance and clustering analysis of metabolomics across Pseudomonas aeruginosa

895 strains. A. Logarithm of raw peak area of all metabolites identified by LC-MS (Liquid

896 chromatography-Mass spectrometry). The metabolites in red were used as internal standard in the data

897 normalization step but removed from the dataset afterwards. B. Hierarchical clustering of the 898 metabolic profiles using Euclidean distance and Ward aggregation method. Each row represents one 899 metabolite and each column represents a specific sample (both strain and sample number are 900 indicated).

A

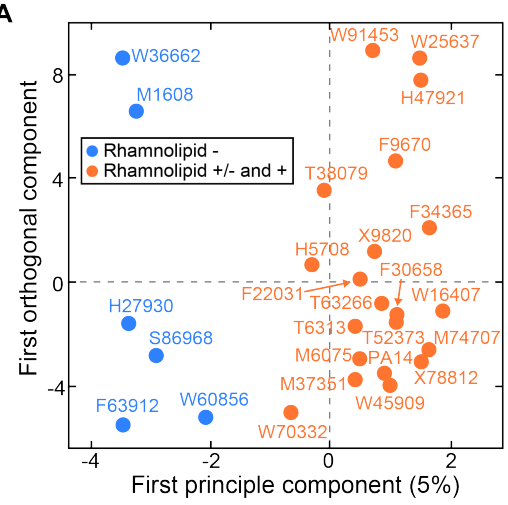

C

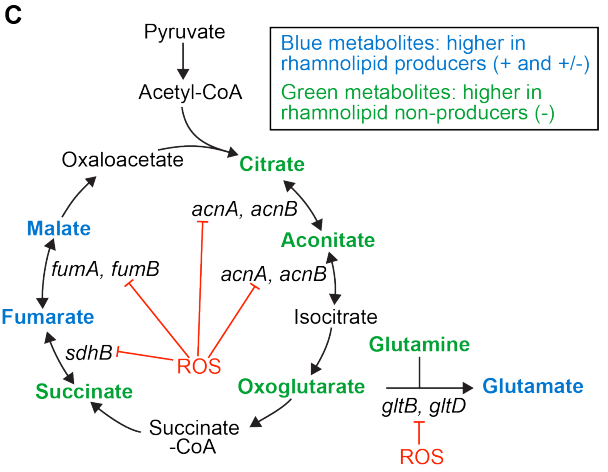

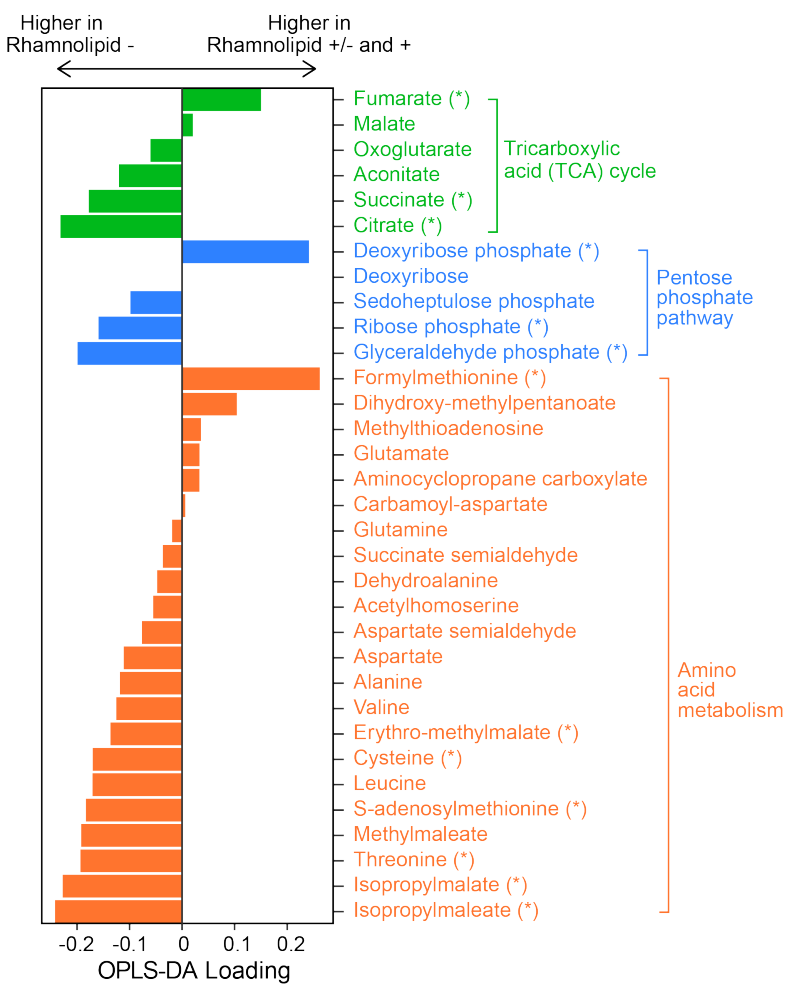

902 Figure 4. Comparative metabolomics between rhamnolipid producers (include both mild and

903 strong production levels) and non-producers. A. The scores plot of the OPLS-DA (orthogonal

904 partial least squares discriminant analysis) model. The first principle component separated the

905 rhamnolipid non-producers from producers. B. OPLS-DA loading values for the first principle

906 component of a selected number of metabolites. Metabolites with asterisk $\left(^{*}\right)$ are significantly

907 different between producers and non-producers (adjusted p-value of a Mann-Whitney test $<0.05$ ). C.

908 Differential abundance of metabolites involved in reactions catalyzed by some Fe-S-containing 909 enzymes whose activities are inhibited by reactive oxygen species (ROS). Abbreviations: acnA 
bioRxiv preprint doi: https://doi org/10.1101/2020.09 02.280495; this version posted September 3,2020 . The copyright holder for this preprint (which was not certified by peer review) is the author/funder, who has granted bioRxiv a license to display the preprint in perpetuity. It is made available under aCC-BY-ND 4.0 International license.

910 (aconitate hydratase A), $a c n B$ (aconitate hydratase B), $s d h B$ (succinate dehydrogenase subunit), fumA

911 (fumarase A), fumB (fumarase B), gltB (glutamate synthase subunit), gltD (glutamate synthase

912 subunit).
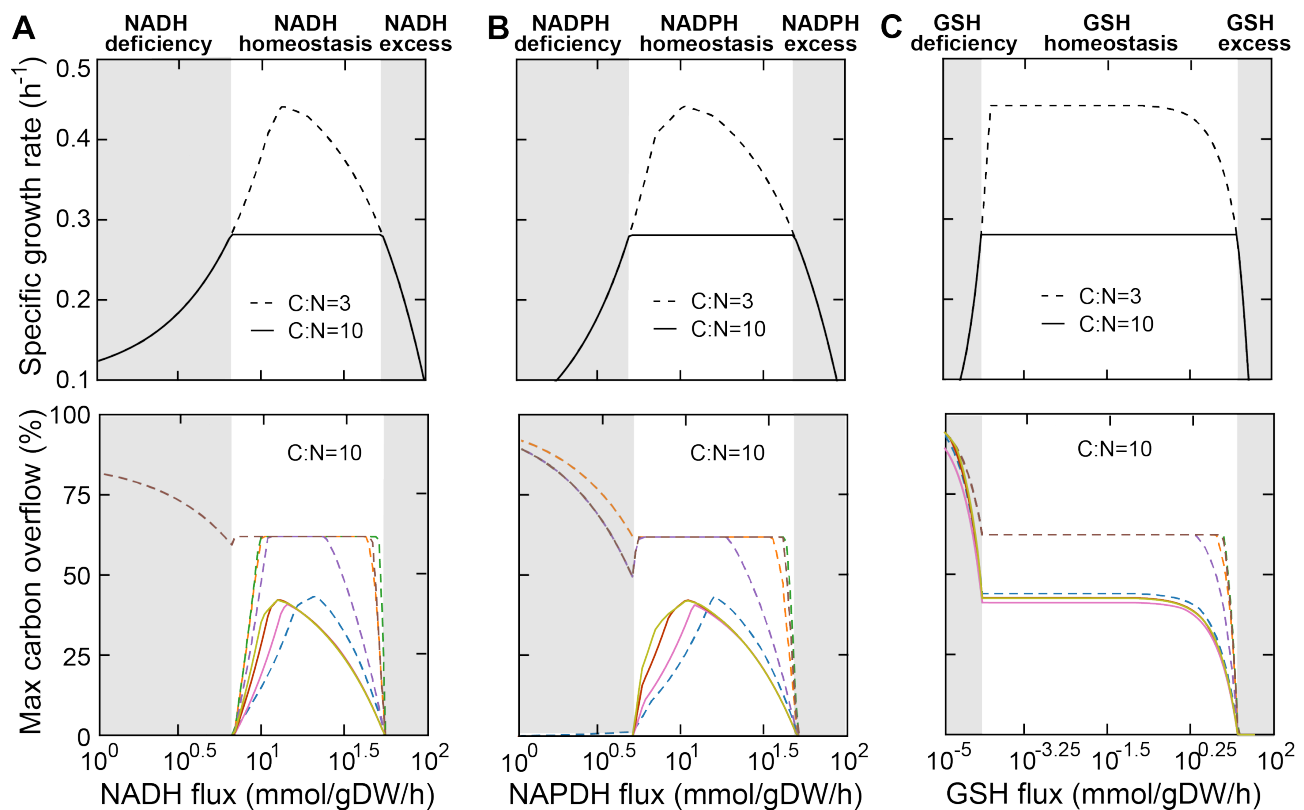

- HAA - monoRL - diRL -- acetate -- aKG --(iso)citrate -- D-lactate --pyruvate

915 Figure 5 Growth and rhamnolipid secretion are constrained by redox status. The redox stress levels

916 are perturbed by altering fluxes of NADH (A. reduced nicotinamide adenine dinucleotide) NADPH

917 (B. reduced nicotinamide adenine dinucleotide phosphate) and GSH (C. reduced glutathione). Upper

918 panels are predicted maximum growth rates and lower panels are predicted maximum byproduct

919 secretion fluxes. C:N indicates the carbon-to-nitrogen ratio between glycerol and ammonium in the

920 culture medium. $\mathrm{C}: \mathrm{N}=3$ and $\mathrm{C}: \mathrm{N}=10$ represent carbon- and nitrogen-limiting conditions respectively.

921 Abbreviations: HAA: 3-(3-hydroxyalkanoyloxy) alkanoate; monoRL: monorhamnolipid; diRL:

922 dirhamnolipid; aKG: alpha-ketoglutarate. 

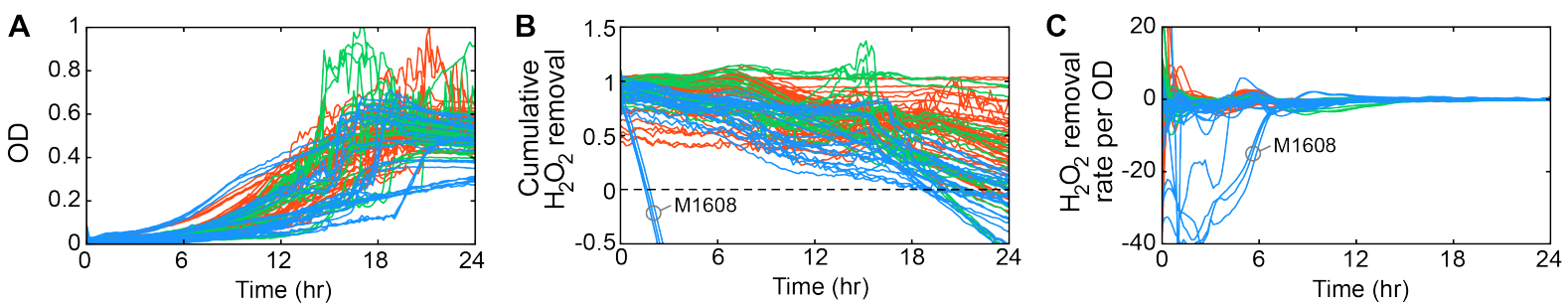

- Rhamnolipid -

- Rhamnolipid +/-

Rhamnolipid +

924 Figure 6. Comparison of the ability of removing culture medium hydrogen peroxide $\left(\mathrm{H}_{2} \mathrm{O}_{2}\right)$ among

925 strong rhamnolipid producers (+), weak producers (+/-), and non-producers (-). A. Population density.

926 B. The total amount of hydrogen peroxide removed from the environment. Negative values indicate

927 net cellular production of hydrogen peroxide released to the environment. C. The specific hydrogen

928 peroxide removal rate. In both $\mathbf{B}$ and $\mathbf{C}$, each trajectory of $\mathrm{H}_{2} \mathrm{O}_{2}$ fluorescence intensity was normalized

929 to the averaged trajectory of the wild-type UCBPP-PA14 strain. 


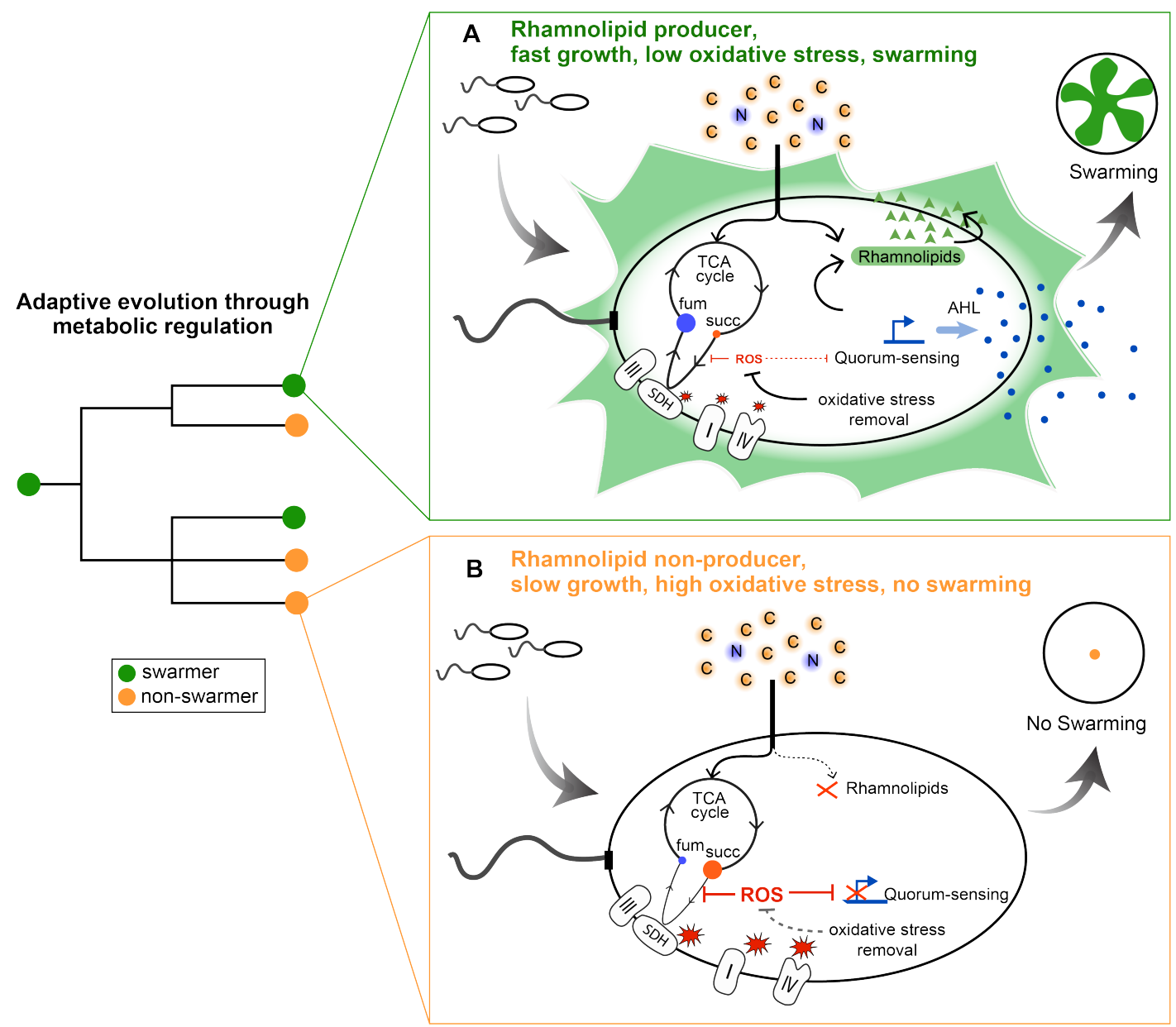

932 Figure 7. Schematic model showing how metabolism regulates evolution of swarming and

933 rhamnolipid production in $P$. aeruginosa. During aerobic growth, cells rely on the TCA cycle and

934 electron transport chain for central energy metabolism, which generates ROS (reactive oxygen

935 species) as byproduct. Particularly, the membrane-located succinate dehydrogenase (SDH), which

936 oxidizes succinate (succ in short, red dot, lower in rhamnolipid producers) to fumarate (fum in short,

937 blue dot, higher in rhamnolipid producers), is an important site of ROS production and serves as the

938 bottleneck of TCA cycle when ROS is excessive. A. Rhamnolipid producers are able to effectively

939 remove ROS; therefore, their TCA cycle fluxes are only minimally inhibited by ROS and remain high

940 to support fast growth. When cells reach certain density and nitrogen becomes limited, rhamnolipid

941 production is turned on by quorum-sensing. B. Rhamnolipid non-producers ineffectively remove ROS

942 and excessive ROS inhibits SDH, resulting in higher level of succinate and lower level of fumarate

943 compared to rhamnolipid producers. The ROS-mediated inhibition significantly slows down the TCA 
944 cycle and cell growth. The increased ROS level is thought to impair quorum-sensing in non-producers

945 and subsequently abolishes rhamnolipid production under the same culture medium of producers. Our

946 model further suggests that null mutations and entire loss of genes that play important roles in redox

947 stress sensing, signaling and protection drive the observed diversity of swarming and rhamnolipid

948 production phenotypes along evolution. 


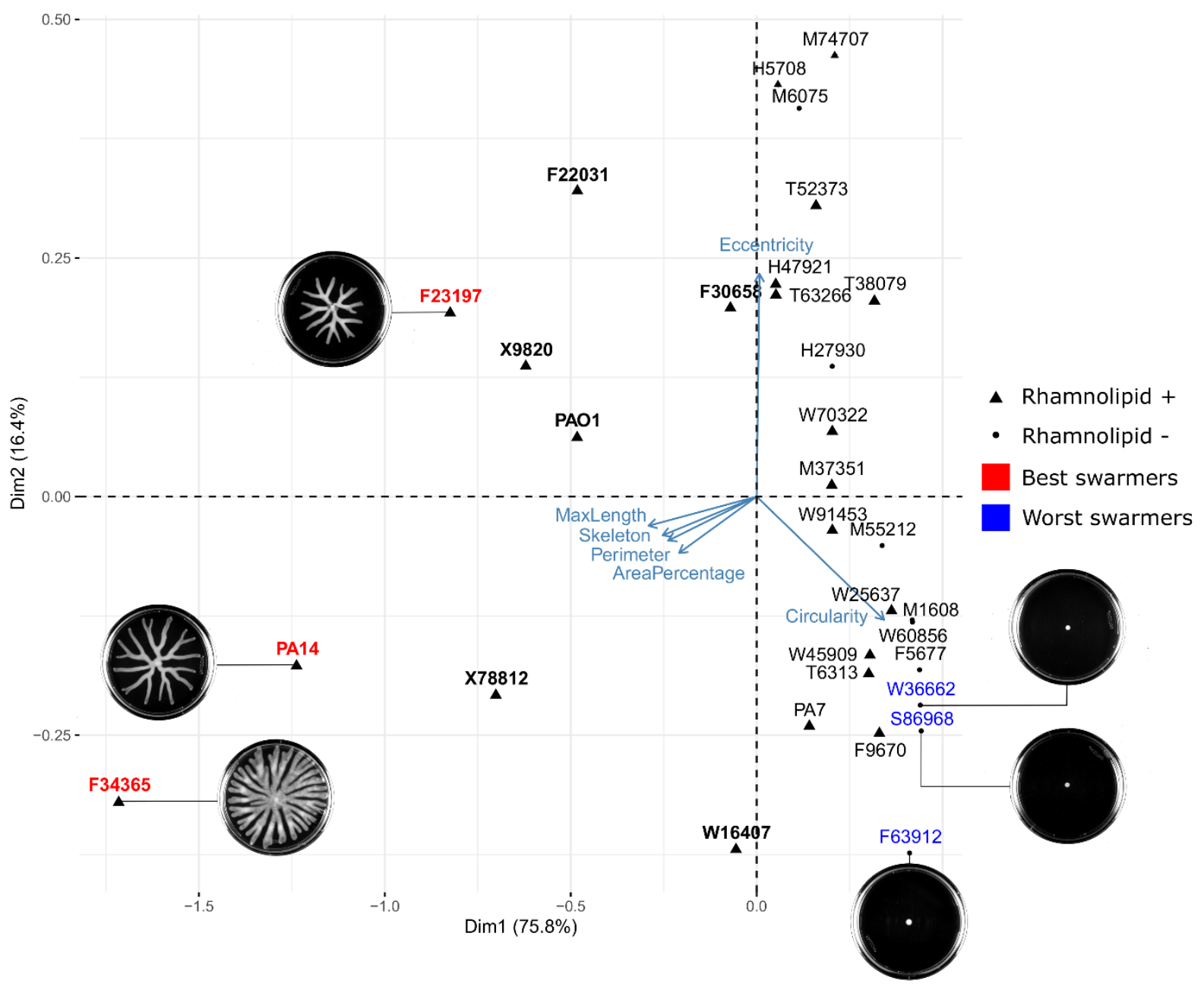

952 Supplementary Figure 1. Principle component analysis and biplot of the swarming phenotype. Six

953 morphological features were extracted from quantitative imaging analysis (see Materials and

954 Methods in the main text), including maximum length (MaxLength), skeleton, perimeter, area of 955 swarming colony (AreaPercentage), circularity and eccentricity. The MaxLength and circularity were 956 the top two features that separate strong swarmers (bold font-weight) from weak swarmers (normal 957 font-weight). The top 3 strains that have visually the largest (best swarmers) and smallest (worst 958 swarmers) swarming colonies are highlighted in red and blue respectively with their swarming images 
bioRxiv preprint doi: https://doi org/10.1101/2020.09.02 280495; this version posted September 3,2020 . The copyright holder for this preprint (which was not certified by peer review) is the author/funder, who has granted bioRxiv a license to display the preprint in perpetuity. It is made available under aCC-BY-ND 4.0 International license.

959 shown alongside. The abilities of these strains to produce rhamnolipids are also indicated by triangles

960 (producers) and dots (non-producers).

A rhamnolipids production

- RL-

$\circ \mathrm{RL}+$
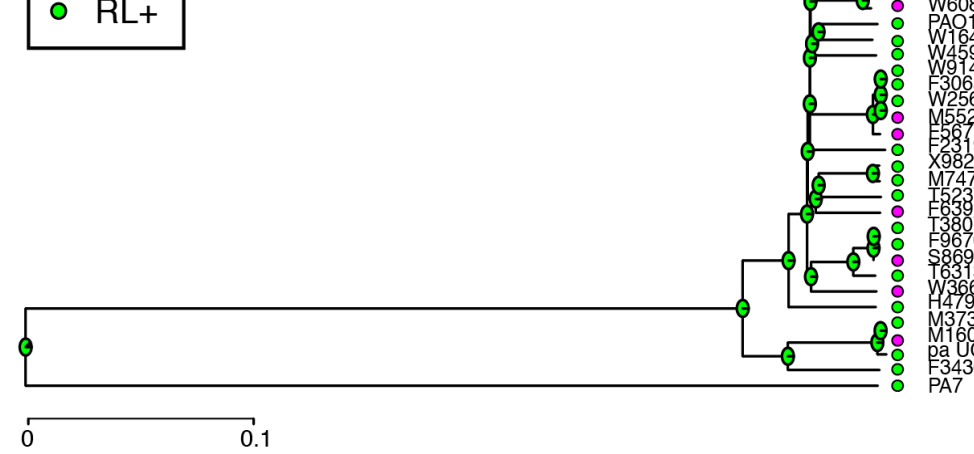

B

swarming

- swarm-

- swarm+

961

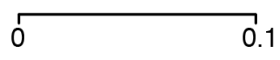

962 Supplementary Figure 2. Phylogenetic ancestor state reconstruction of rhamnolipid production (A)

963 and swarming phenotypes (B). For both (A) and (B), pie charts at the ancestor nodes of branches

964 represent relative likelihood proportion of each possible phenotypic state. We rooted the tree with

965 PA7, a $P$. aeruginosa isolate that is often used as an outlier to root phylogenetic trees. RL,

966 rhamnolipid production. 


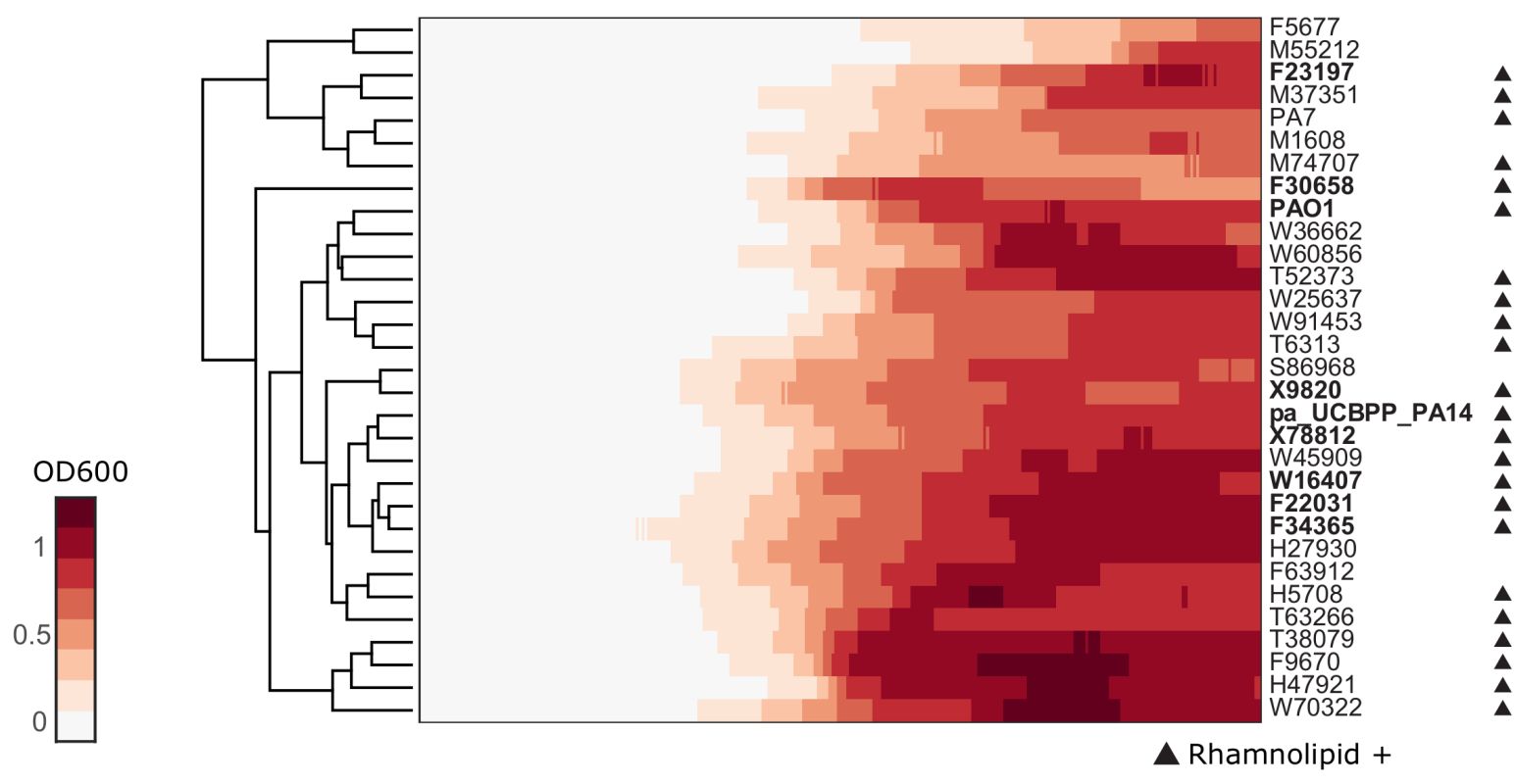

968 Supplementary Figure 3. Clustergram of growth curves of $P$. aeruginosa clinical isolates and three

969 type strains PA14, PAO1 and PA7 in glycerol minimal medium. Euclidean distance was used as the

970 measure of similarity. The strains that are able to swarm are indicated in bold. Most of the swarmers

971 are clustered together. The swarmers that do not fall into this cluster include F30658 and PAO1, both

972 of which are mild swarmers, as well as F23197 whose growth curve has a relatively longer lag time. 

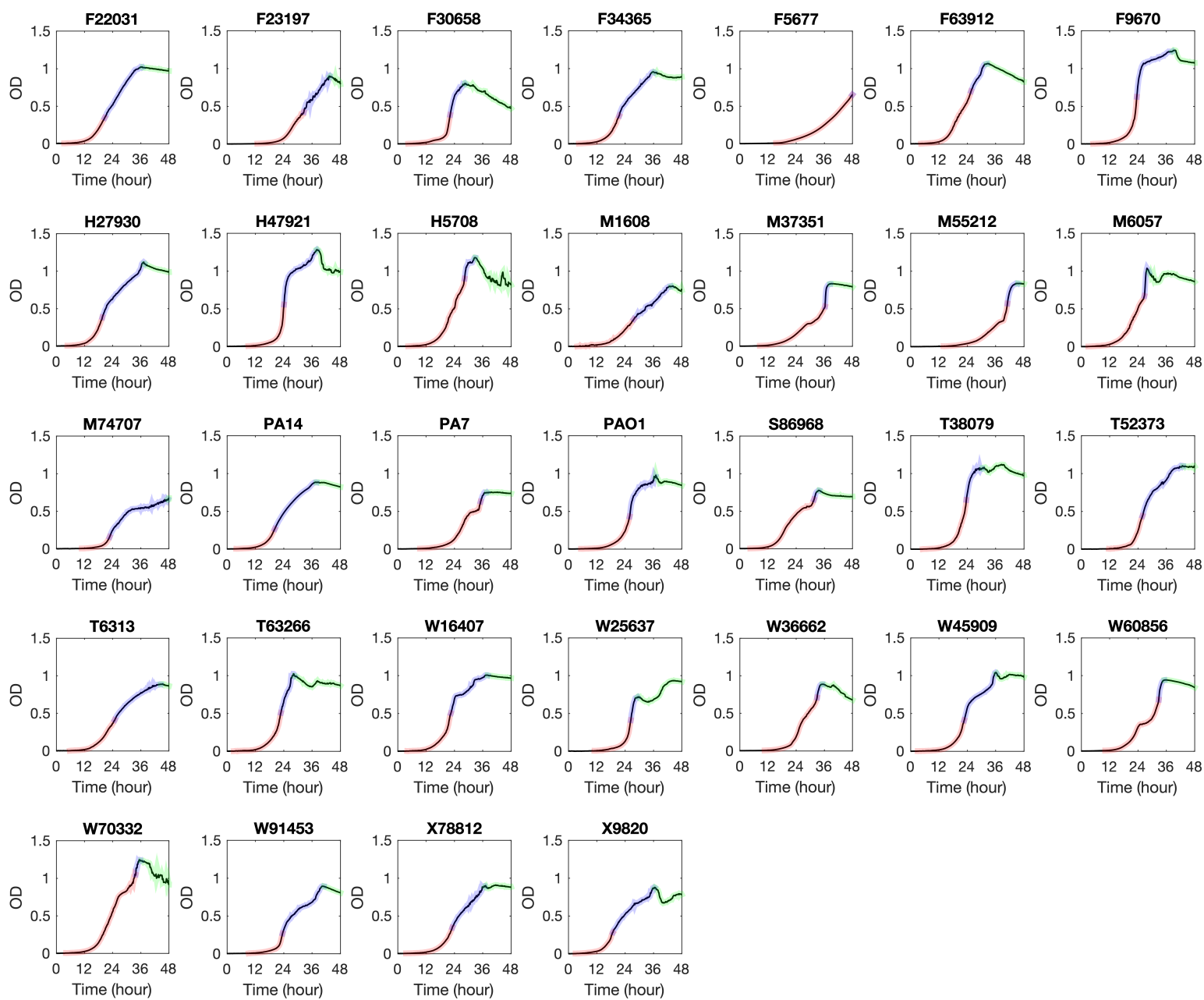

974 Supplementary Figure 4. Growth curve of $P$. aeruginosa strains in glycerol minimal medium. Phase

975 I, II, and III are colored by red, blue and green respectively. 
bioRxiv preprint doi: https://doi org/10.1101/2020.09.02 280495; this version posted September 3, 2020. The copyright holder for this preprint (which was not certified by peer review) is the author/funder, who has granted bioRxiv a license to display the preprint in perpetuity. It is made available under aCC-BY-ND 4.0 International license.

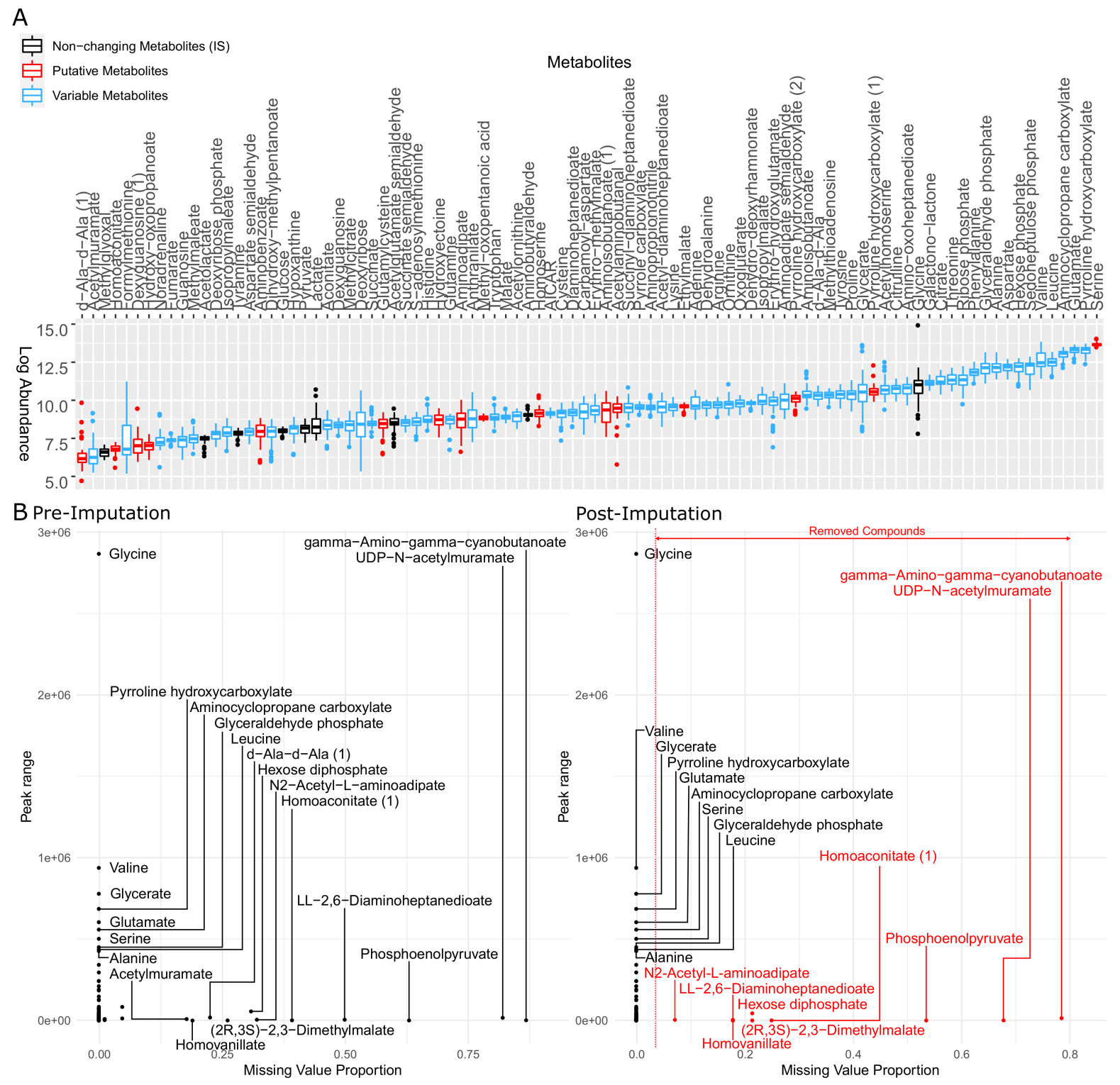

Supplementary Figure S5. A. The same as Fig. 3A of the main text but additionally includes metabolites with putative molecular identifications. These putative metabolites were initially included in clustering metabolomics but later removed from the heatmap (Fig. 3B of the main text). B.

981 Metabolite peak area before (left panel) and after (right panel) imputation. The missing value 982 proportion of a metabolite in the $\mathrm{x}$-axis represents the frequency of missing values of the metabolite 983 across all replicates of all strains in our study. The peak range of a metabolite in the y-axis is defined 984 as the maximum peak area minus the minimum peak area among all non-missing values of the same metabolite across all our strains. 


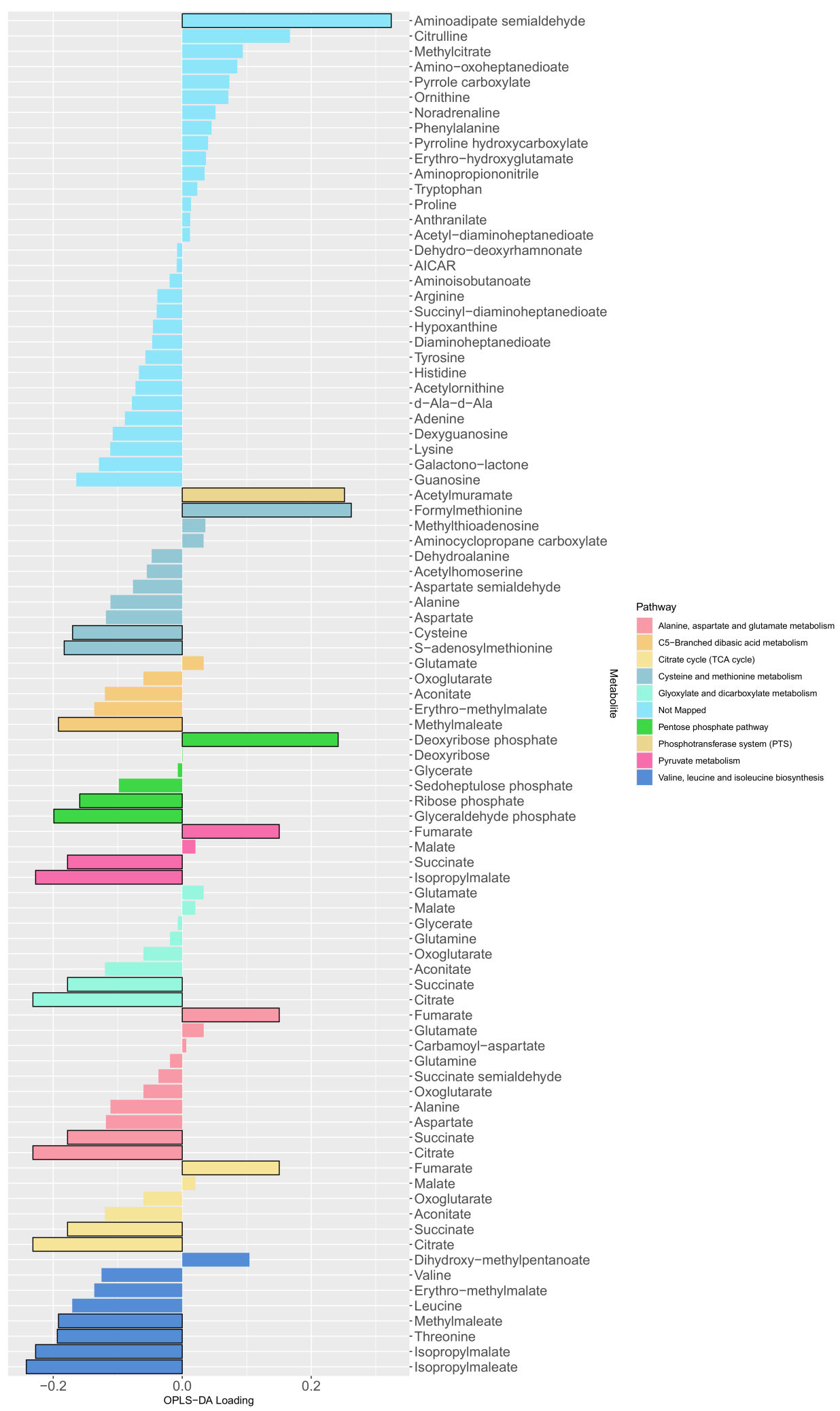

988 Supplementary Figure 6. The loading values of all predictive metabolites of the OPLS-DA model.

989 The differential metabolites between producers and non-producers were determined by a Mann

990 Whitney test (adjusted $p$-values with Benjamini-Hochberg method) with a level of significance of 
bioRxiv preprint doi: https://doi.org/10.1101/2020.09.02.280495; this version posted September 3, 2020. The copyright holder for this preprint (which was not certified by peer review) is the author/funder, who has granted bioRxiv a license to display the preprint in perpetuity. It is made available under aCC-BY-ND 4.0 International license.

9910.05 (bars with black outline) and used as input for a metabolic pathway enrichment with FELLA

992 algorithm. The colors indicate the mapped metabolite pathway for each metabolite. 


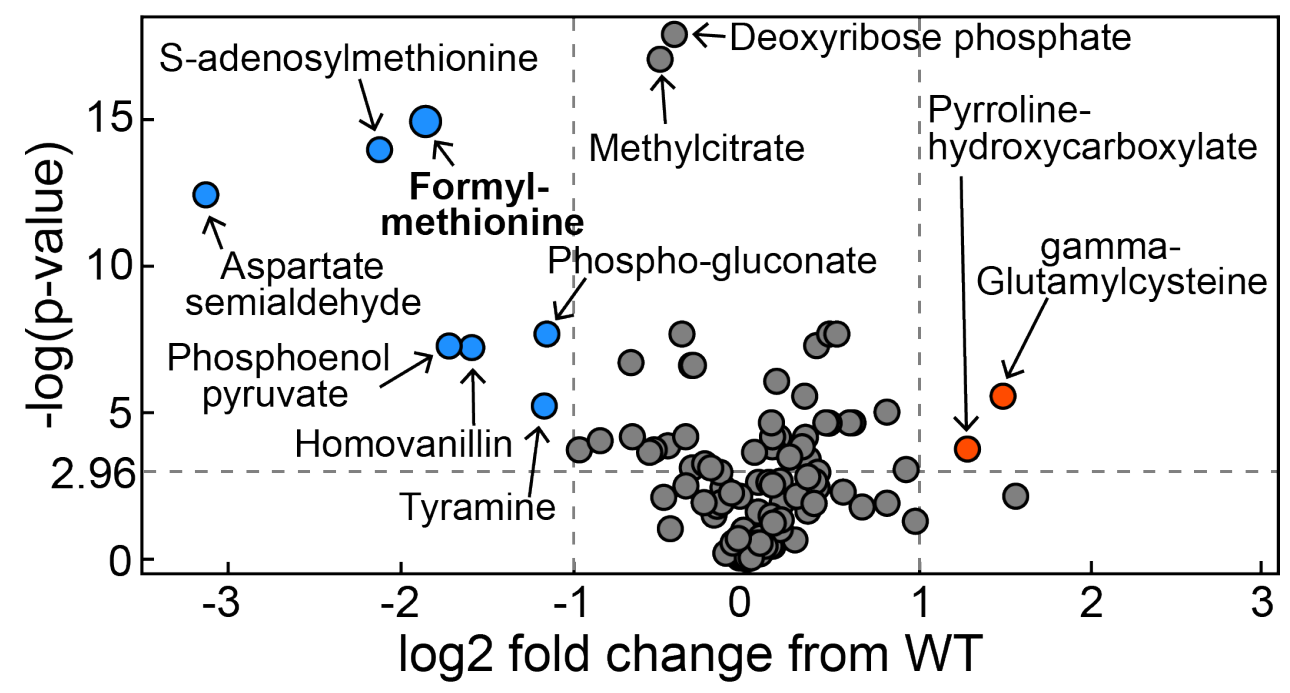

994 Supplementary Figure 7. Volcano plot of metabolomics data between wild-type $P$. aeruginosa

995 UCBPP-PA14 strain and its rhlA mutant grown in glycerol minimal medium (replotted with permission from (Boyle et al, 2017)). 


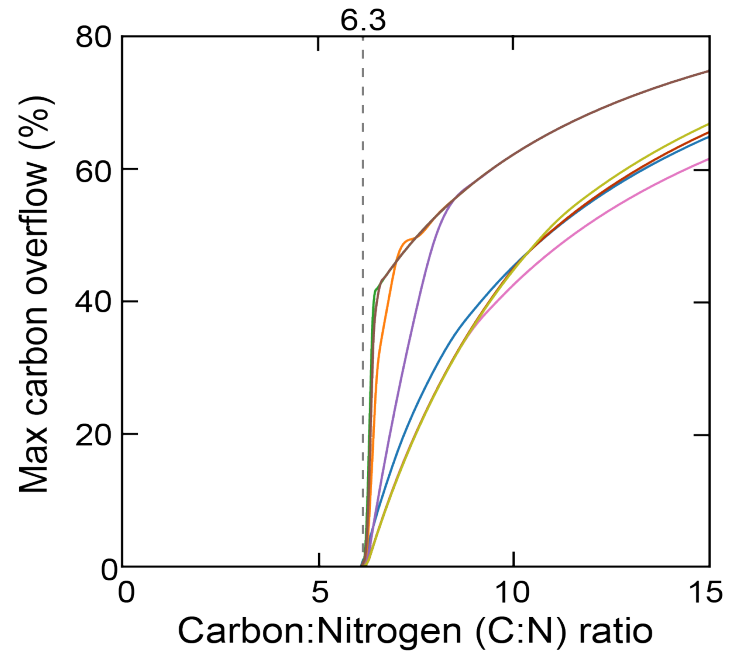

- HAA - monoRL - diRL - acetate -aKG -(iso)citrate -D-lactate - pyruvate

998 Supplementary Figure 8. Theoretical estimation of threshold carbon (glycerol):nitrogen

999 (ammonium) ratio above which carbon is in excess in the sense that carbon release through

1000 rhamnolipids and central carbon metabolites does not compromise biomass production.

1001 Abbreviations: HAA: 3-(3-hydroxyalkanoyloxy)alkanoate; monoRL: monorhamnolipid; diRL:

1002 dirhamnolipid; aKG: alpha-ketoglutarate. 
bioRxiv preprint doi: https://doi.org/10.1101/2020.09.02.280495; this version posted September 3, 2020. The copyright holder for this preprint (which was not certified by peer review) is the author/funder, who has granted bioRxiv a license to display the preprint in perpetuity. It is made available under aCC-BY-ND 4.0 International license.
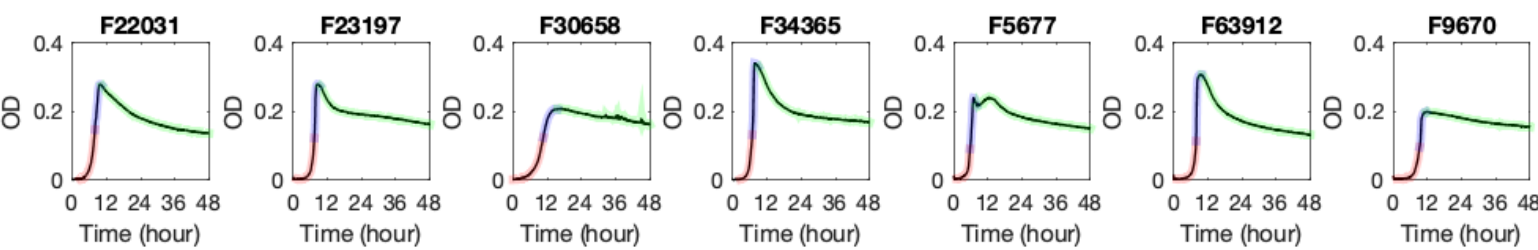

H27930
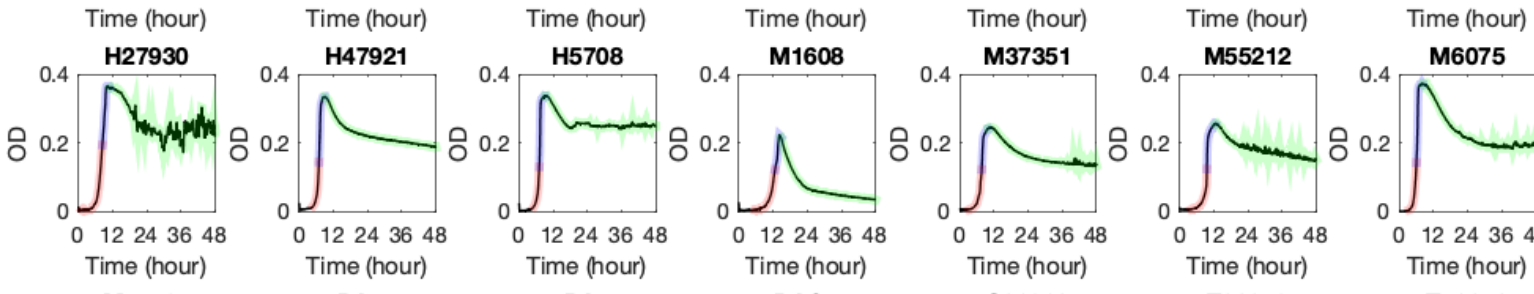

PA14
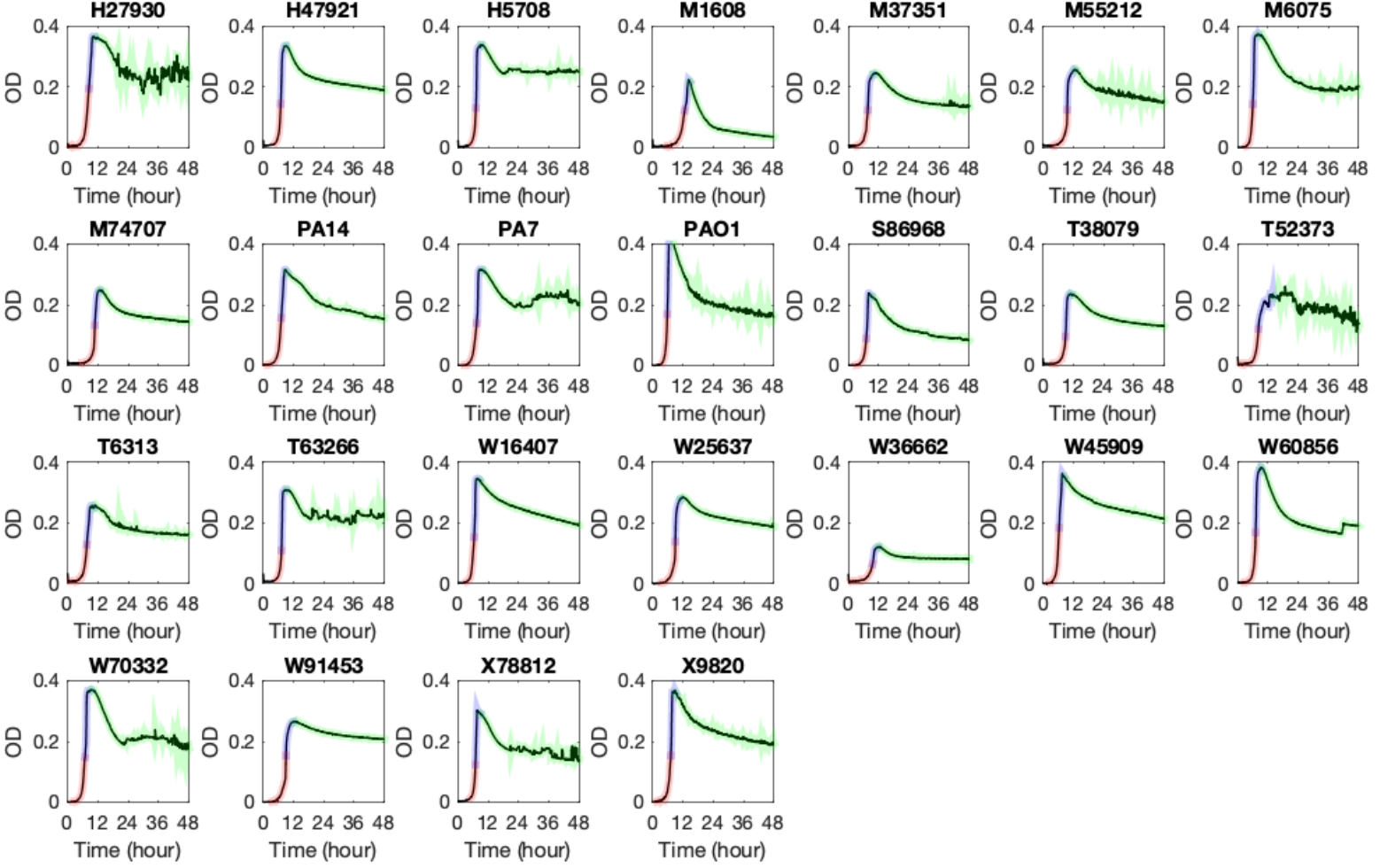

1005 Supplementary Figure 9. Growth curve of Pseudomonas aeruginosa strains in succinate minimal medium. Phase I, II, and III are colored by red, blue and green respectively. 
Phase 1
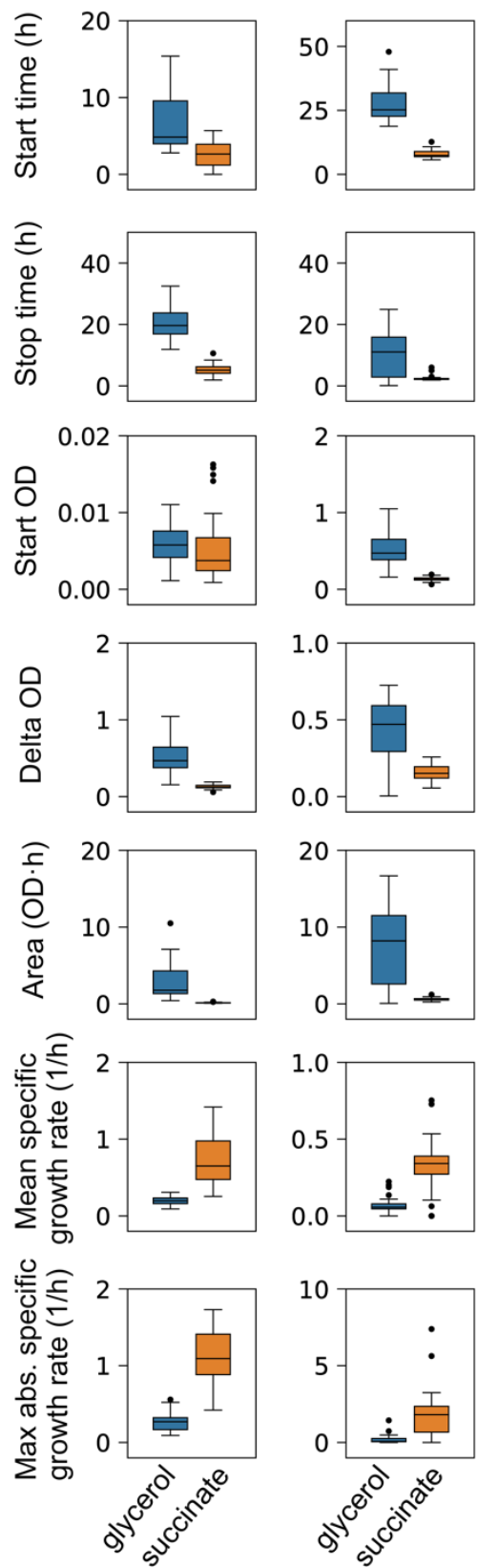
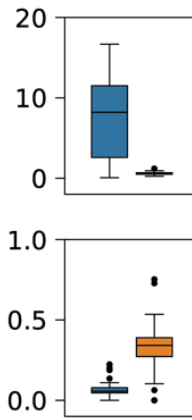

Phase 2

Phase 3
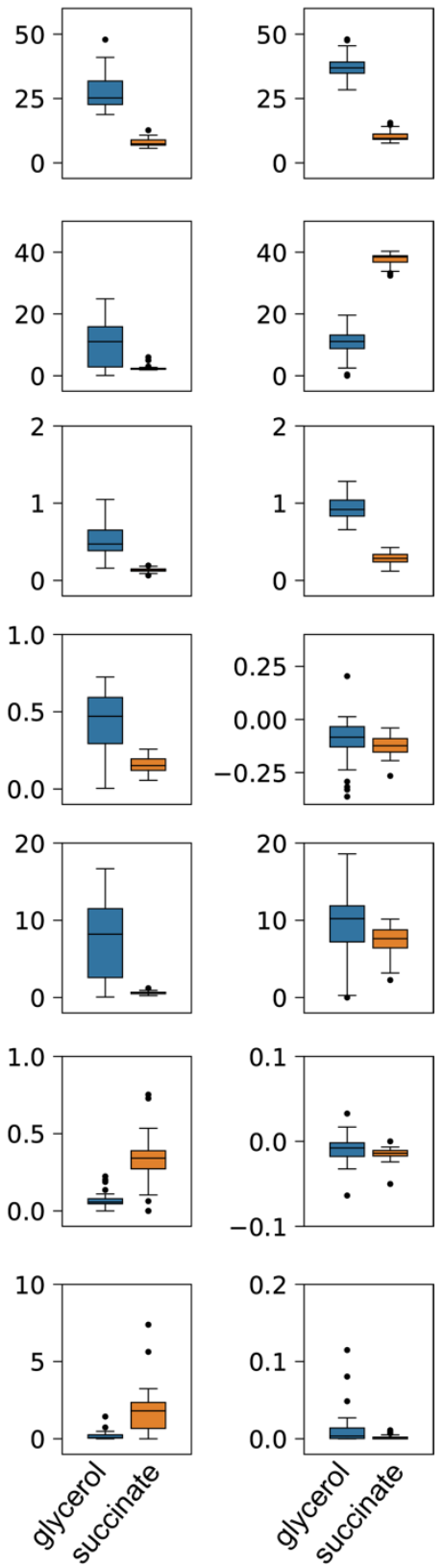

1008 Supplementary Figure 10. Comparison of growth curve features of Pseudomonas aeruginosa strains 1009 grown in glycerol and succinate minimal medium. 
1010 Supplementary Data

1011 Supplementary Table 1: output of the FELLA algorithm. A set of differential compounds was

1012 determined with a Mann-Whitney $\mathrm{U}$ test between rhamnolipid producers and rhamnolipid non

1013 producers ( $p$-value adjusted with Benjamini-Hochberg method, alpha $=0.05)$. These compounds were

1014 used for applying diffusion algorithms over a graph consisting in all entries for Pseudomonas

1015 aeruginosa strain UCBPP-PA14 in KEGG database. The entries shown in the table are the ones with a significative probability of receiving part of the simulated flux.

\begin{tabular}{|c|c|c|c|}
\hline KEGG.id & Entry.type & KEGG.name & p.score \\
\hline pau00020 & pathway & Citrate cycle (TCA cycle) - Pseudomonas aerug... & $2.051 \mathrm{E}-02$ \\
\hline & & Pentose phosphate pathway - Pseudomonas & \\
\hline pau00030 & pathway & aerug... & $2.657 \mathrm{E}-04$ \\
\hline pau00250 & pathway & Alanine, aspartate and glutamate metabolism -... & $3.502 \mathrm{E}-02$ \\
\hline & & Cysteine and methionine metabolism - & \\
\hline pau00270 & pathway & Pseudomo... & $3.106 \mathrm{E}-04$ \\
\hline pau00290 & pathway & Valine, leucine and isoleucine biosynthesis -... & $1.000 \mathrm{E}-06$ \\
\hline pau00620 & pathway & Pyruvate metabolism - Pseudomonas aeruginosa ... & $3.766 \mathrm{E}-02$ \\
\hline pau00630 & pathway & Glyoxylate and dicarboxylate metabolism - Pse... & $1.105 \mathrm{E}-02$ \\
\hline pau00660 & pathway & C5-Branched dibasic acid metabolism - Pseudom... & $8.242 \mathrm{E}-03$ \\
\hline pau01503 & pathway & Cationic antimicrobial peptide (CAMP) resista... & $3.891 \mathrm{E}-02$ \\
\hline pau02060 & pathway & Phosphotransferase system (PTS) - Pseudomonas... & $4.222 \mathrm{E}-02$ \\
\hline M00001 & module & Glycolysis (Embden-Meyerhof pathway), glucose... & $1.613 \mathrm{E}-02$ \\
\hline M00002 & module & Glycolysis, core module involving three-carbo... & $4.022 \mathrm{E}-03$ \\
\hline M00003 & module & Gluconeogenesis, oxaloacetate $=>$ fructose-6P & $2.299 \mathrm{E}-03$ \\
\hline
\end{tabular}




\begin{tabular}{|c|c|c|c|}
\hline M00005 & module & PRPP biosynthesis, ribose $5 P=>$ PRPP & $1.000 \mathrm{E}-06$ \\
\hline M00007 & module & Pentose phosphate pathway, non-oxidative phas... & $1.030 \mathrm{E}-04$ \\
\hline M00009 & module & Citrate cycle (TCA cycle, Krebs cycle) & 7.517E-04 \\
\hline M00010 & module & Citrate cycle, first carbon oxidation, oxaloa... & 4.789E-04 \\
\hline M00011 & module & Citrate cycle, second carbon oxidation, 2-oxo... & 8.189E-03 \\
\hline M00012 & module & Glyoxylate cycle & 1.577E-03 \\
\hline M00019 & module & Valine/isoleucine biosynthesis, pyruvate $=>$ v... & $5.143 \mathrm{E}-03$ \\
\hline M00032 & module & Lysine degradation, lysine $=>$ saccharopine $=>\ldots$ & $2.928 \mathrm{E}-04$ \\
\hline M00049 & module & Adenine ribonucleotide biosynthesis, IMP => A... & $3.245 \mathrm{E}-02$ \\
\hline M00149 & module & Succinate dehydrogenase, prokaryotes & $4.051 \mathrm{E}-02$ \\
\hline M00165 & module & Reductive pentose phosphate cycle (Calvin cyc... & $8.362 \mathrm{E}-04$ \\
\hline M00167 & module & Reductive pentose phosphate cycle, glyceralde... & 2.917E-04 \\
\hline M00173 & module & Reductive citrate cycle (Arnon-Buchanan cycle... & $1.377 \mathrm{E}+09$ \\
\hline M00308 & module & Semi-phosphorylative Entner-Doudoroff pathway... & $2.511 \mathrm{E}-02$ \\
\hline M00344 & module & Formaldehyde assimilation, xylulose monophosp... & 5.467E-03 \\
\hline M00374 & module & Dicarboxylate-hydroxybutyrate cycle & $2.552 \mathrm{E}-02$ \\
\hline M00432 & module & Leucine biosynthesis, 2-oxoisovalerate $=>2-0 .$. & $1.000 \mathrm{E}-06$ \\
\hline M00535 & module & Isoleucine biosynthesis, pyruvate => 2-oxobut... & $1.000 \mathrm{E}-06$ \\
\hline M00570 & module & Isoleucine biosynthesis, threonine $=>2$-oxobu... & 8.290E-04 \\
\hline M00620 & module & Incomplete reductive citrate cycle, acetyl-Co... & $1.000 \mathrm{E}-06$ \\
\hline M00633 & module & Semi-phosphorylative Entner-Doudoroff pathway... & $3.830 \mathrm{E}-02$ \\
\hline M00740 & module & Methylaspartate cycle & $4.398 \mathrm{E}-02$ \\
\hline \multirow[t]{2}{*}{ 1.1.1.85 } & enzyme & 3-isopropylmalate dehydrogenase & $1.000 \mathrm{E}-06$ \\
\hline & & glyceraldehyde-3-phosphate dehydrogenase & \\
\hline 1.2.1.12 & enzyme & (pho... & $1.578 \mathrm{E}-03$ \\
\hline
\end{tabular}




\begin{tabular}{|c|c|c|c|}
\hline & & glyceraldehyde-3-phosphate dehydrogenase & \\
\hline 1.2.1.9 & enzyme & (NAD... & 4.815E-04 \\
\hline 1.3.5.1 & enzyme & succinate dehydrogenase & $5.028 \mathrm{E}+08$ \\
\hline 1.3.5.4 & enzyme & fumarate reductase (quinol) & $5.028 \mathrm{E}+08$ \\
\hline 1.4.1.9 & enzyme & leucine dehydrogenase & $1.032 \mathrm{E}-02$ \\
\hline 1.8.4.15 & enzyme & protein dithiol oxidoreductase (disulfide-for... & $3.891 \mathrm{E}-02$ \\
\hline 2.2.1.1 & enzyme & transketolase & $1.838 \mathrm{E}+08$ \\
\hline 2.2.1.2 & enzyme & transaldolase & $5.939 E-04$ \\
\hline 2.3.3.1 & enzyme & citrate (Si)-synthase & 1.000E-06 \\
\hline 2.3.3.13 & enzyme & 2-isopropylmalate synthase & $1.000 \mathrm{E}-06$ \\
\hline 2.4.2.1 & enzyme & purine-nucleoside phosphorylase & $1.000 \mathrm{E}-06$ \\
\hline 2.4.2.2 & enzyme & pyrimidine-nucleoside phosphorylase & $1.000 \mathrm{E}-06$ \\
\hline 2.5.1.49 & enzyme & O-acetylhomoserine aminocarboxypropyltransfer... & 4.137E-02 \\
\hline 2.6.1.2 & enzyme & alanine transaminase & $1.000 \mathrm{E}-06$ \\
\hline 2.6.1.42 & enzyme & branched-chain-amino-acid transaminase & $1.985 \mathrm{E}-02$ \\
\hline 2.6.1.66 & enzyme & valine---pyruvate transaminase & $1.000 \mathrm{E}-06$ \\
\hline 2.7.1.15 & enzyme & ribokinase & $1.791 \mathrm{E}-02$ \\
\hline 2.7.1.221 & enzyme & $\mathrm{N}$-acetylmuramate 1-kinase & $1.000 \mathrm{E}-06$ \\
\hline 2.7.2.3 & enzyme & phosphoglycerate kinase & $1.747 \mathrm{E}-02$ \\
\hline 2.7.6.1 & enzyme & ribose-phosphate diphosphokinase & $1.000 \mathrm{E}-06$ \\
\hline 2.7.9.2 & enzyme & pyruvate, water dikinase & 7.185E-03 \\
\hline 2.8.3.18 & enzyme & succinyl-CoA:acetate CoA-transferase & $1.672 \mathrm{E}-02$ \\
\hline 3.1.3.105 & enzyme & N-acetyl-D-muramate 6-phosphate phosphatase & $1.000 \mathrm{E}-06$ \\
\hline 3.1.3.11 & enzyme & fructose-bisphosphatase & $3.302 \mathrm{E}-02$ \\
\hline 3.1.3.48 & enzyme & protein-tyrosine-phosphatase & 1.524E-02 \\
\hline
\end{tabular}




\begin{tabular}{|c|c|c|c|}
\hline 3.2.2.10 & enzyme & pyrimidine-5'-nucleotide nucleosidase & $1.000 \mathrm{E}-06$ \\
\hline 3.2.2.4 & enzyme & AMP nucleosidase & $1.000 \mathrm{E}-06$ \\
\hline 3.4.21.107 & enzyme & peptidase Do & $1.929 \mathrm{E}-02$ \\
\hline 3.4.24.40 & enzyme & serralysin & 3.891E-02 \\
\hline 3.5.1.28 & enzyme & $\mathrm{N}$-acetylmuramoyl-L-alanine amidase & $1.000 \mathrm{E}-06$ \\
\hline 3.5.4.2 & enzyme & adenine deaminase & $1.786 \mathrm{E}-02$ \\
\hline 3.6.1.13 & enzyme & ADP-ribose diphosphatase & $7.396 \mathrm{E}+08$ \\
\hline 3.7.1.20 & enzyme & 3-fumarylpyruvate hydrolase & 2.495E-02 \\
\hline 4.1.1.31 & enzyme & phosphoenolpyruvate carboxylase & 4.009E-02 \\
\hline 4.1.2.13 & enzyme & fructose-bisphosphate aldolase & $2.966 \mathrm{E}-03$ \\
\hline 4.1.2.14 & enzyme & 2-dehydro-3-deoxy-phosphogluconate aldolase & $2.363 \mathrm{E}-02$ \\
\hline 4.1.2.48 & enzyme & low-specificity L-threonine aldolase & $9.416 \mathrm{E}-04$ \\
\hline 4.1.3.1 & enzyme & isocitrate lyase & $1.814 \mathrm{E}-02$ \\
\hline 4.2.1.2 & enzyme & fumarate hydratase & $1.000 \mathrm{E}-06$ \\
\hline 4.2.1.20 & enzyme & tryptophan synthase & 3.153E-02 \\
\hline 4.2.1.3 & enzyme & aconitate hydratase & $1.000 \mathrm{E}-06$ \\
\hline 4.2.1.33 & enzyme & 3-isopropylmalate dehydratase & $1.000 \mathrm{E}-06$ \\
\hline 4.2.1.35 & enzyme & (R)-2-methylmalate dehydratase & $1.000 \mathrm{E}-06$ \\
\hline 4.2.3.1 & enzyme & threonine synthase & $3.155 E-02$ \\
\hline 4.3.1.1 & enzyme & aspartate ammonia-lyase & $1.000 \mathrm{E}-06$ \\
\hline 4.3.1.19 & enzyme & threonine ammonia-lyase & $1.000 \mathrm{E}-06$ \\
\hline 4.3.2.1 & enzyme & argininosuccinate lyase & 1.647E-02 \\
\hline 4.3.2.2 & enzyme & adenylosuccinate lyase & 9.353E-04 \\
\hline 5.1.3.1 & enzyme & ribulose-phosphate 3-epimerase & $1.624 \mathrm{E}-02$ \\
\hline 5.2.1.8 & enzyme & peptidylprolyl isomerase & $3.891 \mathrm{E}-02$ \\
\hline
\end{tabular}




\begin{tabular}{|c|c|c|c|}
\hline 5.3.1.1 & enzyme & triose-phosphate isomerase & $1.698 \mathrm{E}+08$ \\
\hline 5.3.1.6 & enzyme & ribose-5-phosphate isomerase & $2.338 \mathrm{E}-02$ \\
\hline 5.4.2.2 & enzyme & phosphoglucomutase (alpha-D-glucose-1,6-bisph... & $4.898 \mathrm{E}+09$ \\
\hline 6.2 .1 .5 & enzyme & succinate---CoA ligase (ADP-forming) & $6.932 \mathrm{E}-03$ \\
\hline 6.3 .1 .2 & enzyme & glutamine synthetase & 3.639E-02 \\
\hline 6.4.1.1 & enzyme & pyruvate carboxylase & $4.182 E+07$ \\
\hline R00086 & reaction & ATP phosphohydrolase & 3.936E-02 \\
\hline R00122 & reaction & ADP phosphohydrolase & 3.029E-02 \\
\hline R00177 & reaction & ATP:L-methionine S-adenosyltransferase & $1.894 \mathrm{E}-02$ \\
\hline R00182 & reaction & AMP phosphoribohydrolase & $1.000 \mathrm{E}-06$ \\
\hline R00220 & reaction & L-serine ammonia-lyase & $2.595 \mathrm{E}-02$ \\
\hline R00258 & reaction & L-Alanine:2-oxoglutarate aminotransferase & 4.726E-02 \\
\hline R00351 & reaction & acetyl-CoA:oxaloacetate C-acetyltransferase (... & 1.000E-06 \\
\hline R00352 & reaction & acetyl-CoA:oxaloacetate C-acetyltransferase $[\ldots$ & $1.000 \mathrm{E}-06$ \\
\hline R00402 & reaction & succinate:NAD+ oxidoreductase & $1.000 \mathrm{E}-06$ \\
\hline R00405 & reaction & Succinate:CoA ligase (ADP-forming) & 4.397E+09 \\
\hline R00432 & reaction & Succinate:CoA ligase (GDP-forming) & $2.166 \mathrm{E}-02$ \\
\hline R00446 & reaction & L-lysine:NAD+ 6-oxidoreductase (deaminating) & 1.000E-06 \\
\hline R00479 & reaction & isocitrate glyoxylate-lyase (succinate-formin... & $3.522 \mathrm{E}-02$ \\
\hline R00490 & reaction & L-aspartate ammonia-lyase (fumarate-forming) & $1.000 \mathrm{E}-06$ \\
\hline R00510 & reaction & cytidine-5'-monophosphate phosphoribohydrolas... & $1.000 \mathrm{E}-06$ \\
\hline R00653 & reaction & $\mathrm{N}$-formyl-L-methionine amidohydrolase & $1.000 \mathrm{E}-06$ \\
\hline R00751 & reaction & L-threonine acetaldehyde-lyase (glycine-formi... & $1.000 \mathrm{E}-06$ \\
\hline R00782 & reaction & L-cysteine hydrogen-sulfide-lyase (deaminatin... & $1.000 \mathrm{E}-06$ \\
\hline R00891 & reaction & L-serine hydro-lyase (adding hydrogen sulfide... & $1.000 \mathrm{E}-06$ \\
\hline
\end{tabular}




\begin{tabular}{|c|c|c|c|}
\hline R00892 & reaction & L-cysteine:NAD+ oxidoreductase & $1.384 \mathrm{E}-02$ \\
\hline R00893 & reaction & L-Cysteine:oxygen oxidoreductase & 7.899E-03 \\
\hline R00894 & reaction & L-glutamate:L-cysteine gamma-ligase (ADP-form... & $3.659 \mathrm{E}-03$ \\
\hline R00895 & reaction & L-Cysteine:2-oxoglutarate aminotransferase & $1.786 \mathrm{E}-02$ \\
\hline R00897 & reaction & O3-acetyl-L-serine:hydrogen-sulfide 2 -amino-2... & 4.683E-03 \\
\hline R00901 & reaction & L-cysteine hydrogen-sulfide-lyase (adding sul... & 4.539E-03 \\
\hline R00994 & reaction & $(2 \mathrm{R}, 3 \mathrm{~S})-3-$ methylmalate:NAD+ oxidoreductase & $1.000 \mathrm{E}-06$ \\
\hline R00996 & reaction & L-threonine ammonia-lyase (2-oxobutanoate-for... & $1.000 \mathrm{E}-06$ \\
\hline R00999 & reaction & O-Succinyl-L-homoserine succinate-lyase (deam... & $2.925 \mathrm{E}-02$ \\
\hline R01001 & reaction & L-cystathionine cysteine-lyase (deaminating & 3.769E-04 \\
\hline R01015 & reaction & D-glyceraldehyde-3-phosphate aldose-ketose-is... & $1.000 \mathrm{E}-06$ \\
\hline R01049 & reaction & ATP:D-ribose-5-phosphate diphosphotransferase & 1.000E-06 \\
\hline R01051 & reaction & ATP:D-ribose 5-phosphotransferase & 1.000E-06 \\
\hline R01053 & reaction & Ribose-5-phosphate:ammonia ligase (ADP-formin... & $1.804 \mathrm{E}+07$ \\
\hline R01054 & reaction & ADP-ribose ribophosphohydrolase & $9.884 \mathrm{E}+08$ \\
\hline R01055 & reaction & uracil hydro-lyase (adding D-ribose 5-phospha... & $2.245 \mathrm{E}-03$ \\
\hline R01056 & reaction & D-ribose-5-phosphate aldose-ketose-isomerase & $1.000 \mathrm{E}-06$ \\
\hline \multirow[t]{2}{*}{ R01057 } & reaction & D-Ribose 1,5-phosphomutase & $1.000 \mathrm{E}-06$ \\
\hline & & D-glyceraldehyde 3-phosphate:NADP+ & \\
\hline R01058 & reaction & oxidoreduc... & $1.000 \mathrm{E}-06$ \\
\hline \multirow[t]{2}{*}{ R01059 } & reaction & ATP:D-glyceraldehyde 3-phosphotransferase & 1.000E-06 \\
\hline & & D-glyceraldehyde-3-phosphate:NAD+ & \\
\hline \multirow[t]{2}{*}{ R01061 } & reaction & oxidoreduct... & $1.357 \mathrm{E}-04$ \\
\hline & & D-glyceraldehyde-3-phosphate:NADP+ & \\
\hline R01063 & reaction & oxidoreduc... & $1.690 \mathrm{E}-04$ \\
\hline
\end{tabular}




\begin{tabular}{|c|c|c|c|}
\hline R01066 & reaction & 2-deoxy-D-ribose-5-phosphate acetaldehyde-lya... & 1.494E-04 \\
\hline R01067 & reaction & D-Fructose 6-phosphate:D-glyceraldehyde-3-pho... & $5.048 \mathrm{E}+08$ \\
\hline R01068 & reaction & D-fructose-1,6-bisphosphate D-glyceraldehyde-... & $8.170 E+09$ \\
\hline R01069 & reaction & D-tagatose 1,6-bisphosphate D-glyceraldehyde-... & 4.029E-04 \\
\hline R01070 & reaction & beta-D-fructose-1,6-bisphosphate D-glyceralde... & $8.852 E+08$ \\
\hline R01082 & reaction & (S)-malate hydro-lyase (fumarate-forming) & $1.000 \mathrm{E}-06$ \\
\hline R01083 & reaction & N6-(1,2-dicarboxyethyl)AMP AMP-lyase (fumarat... & $2.469 \mathrm{E}-03$ \\
\hline R01085 & reaction & 3-fumarylpyruvate fumarylhydrolase & $1.563 \mathrm{E}-02$ \\
\hline R01086 & reaction & 2-(Nomega-L-arginino)succinate arginine-lyase... & $5.126 \mathrm{E}-03$ \\
\hline R01087 & reaction & Maleate cis-trans-isomerase & $2.433 E+09$ \\
\hline R01113 & reaction & Glutathione:L-cystine oxidoreductase & 3.593E-02 \\
\hline R01128 & reaction & 5'-Inosinate phosphoribohydrolase & $1.000 \mathrm{E}-06$ \\
\hline R01213 & reaction & acetyl-CoA:3-methyl-2-oxobutanoate C-acetyltr... & $1.000 \mathrm{E}-06$ \\
\hline R01215 & reaction & L-Valine:pyruvate aminotransferase & $3.535 \mathrm{E}-02$ \\
\hline R01244 & reaction & Adenine aminohydrolase & $2.846 \mathrm{E}-02$ \\
\hline R01270 & reaction & Nicotinamide D-ribonucleotide phosphoribohydr... & $1.000 \mathrm{E}-06$ \\
\hline R01322 & reaction & Citrate:CoA ligase (ADP-forming) & $3.624 \mathrm{E}-04$ \\
\hline R01324 & reaction & citrate hydroxymutase & $1.000 \mathrm{E}-06$ \\
\hline R01325 & reaction & citrate hydro-lyase (cis-aconitate-forming) & $1.000 \mathrm{E}-06$ \\
\hline R01364 & reaction & 4-fumarylacetoacetate fumarylhydrolase & 2.477E-02 \\
\hline R01440 & reaction & D-Xylulose-5-phosphate:formaldehyde glycolald... & $4.322 E+09$ \\
\hline R01465 & reaction & L-threonine:NAD+ oxidoreductase & $1.000 \mathrm{E}-06$ \\
\hline R01466 & reaction & O-phospho-L-homoserine phosphate-lyase (addin... & $6.537 E+09$ \\
\hline R01561 & reaction & adenosine:phosphate alpha-D-ribosyltransferas... & $2.961 \mathrm{E}-03$ \\
\hline R01570 & reaction & thymidine:phosphate deoxy-alpha-D-ribosyltran... & $1.000 \mathrm{E}-06$ \\
\hline
\end{tabular}




\begin{tabular}{|c|c|c|c|}
\hline R01621 & reaction & D-xylulose 5-phosphate D-glyceraldehyde-3-pho... & $1.000 \mathrm{E}-06$ \\
\hline R01641 & reaction & sedoheptulose-7-phosphate:D-glyceraldehyde-3-... & 1.000E-06 \\
\hline R01652 & reaction & 4-Methyl-2-oxopentanoate + CO2 <=> (2S)-2-Iso... & 4.391E-03 \\
\hline R01827 & reaction & sedoheptulose-7-phosphate:D-glyceraldehyde-3-... & 1.594E-04 \\
\hline R01830 & reaction & beta-D-Fructose 6-phosphate:D-glyceraldehyde-... & $3.088 \mathrm{E}+08$ \\
\hline R01863 & reaction & inosine:phosphate alpha-D-ribosyltransferase & 7.366E-03 \\
\hline R01867 & reaction & (S)-dihydroorotate:fumarate oxidoreductase & $1.035 \mathrm{E}-03$ \\
\hline R01876 & reaction & uridine:phosphate alpha-D-ribosyltransferase & $2.008 \mathrm{E}-03$ \\
\hline R01969 & reaction & Deoxyguanosine:orthophosphate ribosyltransfer... & $1.000 E-06$ \\
\hline R02147 & reaction & guanosine:phosphate alpha-D-ribosyltransferas... & $1.837 \mathrm{E}-02$ \\
\hline R02164 & reaction & succinate:quinone oxidoreductase & $1.000 \mathrm{E}-06$ \\
\hline R02294 & reaction & N-Ribosylnicotinamide:orthophosphate ribosylt... & 4.332E-02 \\
\hline R02296 & reaction & cytidine:orthophosphate alpha-D-ribosyltransf... & 3.703E-02 \\
\hline R02297 & reaction & Xanthosine:orthophosphate ribosyltransferase & 4.035E-02 \\
\hline R02313 & reaction & N6-(L-1,3-Dicarboxypropyl)-L-lysine:NAD+ oxid... & $1.000 \mathrm{E}-06$ \\
\hline R02315 & reaction & N6-(L-1,3-Dicarboxypropyl)-L-lysine:NADP+ oxi... & $1.000 \mathrm{E}-06$ \\
\hline R02317 & reaction & (S)-2-amino-6-oxohexanoate hydro-lyase (spont... & $1.000 \mathrm{E}-06$ \\
\hline R02340 & reaction & (1S,2R)-1-C-(indol-3-yl)glycerol 3-phosphate ... & 7.357E-03 \\
\hline R02409 & reaction & Coenzyme A:oxidized-glutathione oxidoreductas... & $4.474 \mathrm{E}-02$ \\
\hline R02484 & reaction & deoxyuridine:orthophosphate 2-deoxy-D-ribosyl... & $1.000 E-06$ \\
\hline R02557 & reaction & Deoxyadenosine:orthophosphate ribosyltransfer... & $1.000 E-06$ \\
\hline R02722 & reaction & L-serine hydro-lyase [adding 1-C-(indol-3-yl)... & $3.011 E-03$ \\
\hline \multirow[t]{2}{*}{ R02748 } & reaction & Deoxyinosine:orthophosphate ribosyltransferas... & $1.000 \mathrm{E}-06$ \\
\hline & & 2-deoxy-D-ribose 1-phosphate 1,5- & \\
\hline R02749 & reaction & phosphomutas... & $1.000 E-06$ \\
\hline
\end{tabular}




\begin{tabular}{|c|c|c|c|}
\hline \multirow[b]{2}{*}{ R03102 } & \multirow[b]{2}{*}{ reaction } & \multicolumn{2}{|l|}{ L-2-aminoadipate-6-semialdehyde:NAD+6- } \\
\hline & & oxidor... & $1.000 \mathrm{E}-06$ \\
\hline & & L-2-aminoadipate-6-semialdehyde:NADP+6- & \\
\hline R03103 & reaction & oxido... & $1.000 \mathrm{E}-06$ \\
\hline R03217 & reaction & O-Acetyl-L-homoserine succinate-lyase (adding... & $3.059 \mathrm{E}-02$ \\
\hline $\mathrm{R} 03260$ & reaction & O-Succinyl-L-homoserine succinate-lyase (addi... & $2.438 \mathrm{E}-03$ \\
\hline R03524 & reaction & L-cysteine hydrogen-sulfide-lyase (adding HCN... & $3.504 \mathrm{E}-02$ \\
\hline R03896 & reaction & (R)-2-Methylmalate hydro-lyase (2-methylmalea... & $1.149 E+08$ \\
\hline R03898 & reaction & 2-methylmaleate hydratase & $1.000 \mathrm{E}-06$ \\
\hline R03968 & reaction & 2-Isopropylmalate hydro-lyase & $1.000 \mathrm{E}-06$ \\
\hline \multirow[t]{2}{*}{ R04001 } & reaction & 3-Isopropylmalate hydro-lyase & $1.000 \mathrm{E}-06$ \\
\hline & & L-2-aminoadipate-6-semialdehyde:NADP+6- & \\
\hline R04390 & reaction & oxido... & 2.104E-02 \\
\hline R04426 & reaction & (2R,3S)-3-isopropylmalate:NAD+ oxidoreductase & $1.000 \mathrm{E}-06$ \\
\hline R04559 & reaction & 1-(5'-Phosphoribosyl)-5-amino-4-(N-succinocar... & $7.225 \mathrm{E}-03$ \\
\hline R05598 & reaction & Benzylsuccinate fumarate-lyase & $1.083 \mathrm{E}-02$ \\
\hline R05605 & reaction & 2-dehydro-3-deoxy-6-phospho-D-gluconate D-gly... & $2.199 \mathrm{E}-03$ \\
\hline R05636 & reaction & 1-Deoxy-D-xylulose-5-phosphate pyruvate-lyase... & $3.417 \mathrm{E}-03$ \\
\hline R06531 & reaction & ATP:L-threonine O-phosphotransferase & $2.361 \mathrm{E}-04$ \\
\hline R06590 & reaction & sedoheptulose-7-phosphate:D-glyceraldehyde-3-... & $1.654 \mathrm{E}-02$ \\
\hline \multirow[t]{2}{*}{ R06789 } & reaction & (2Z,4E,7E)-2-Hydroxy-6-oxonona-2,4,7-triene-1... & $1.163 \mathrm{E}-02$ \\
\hline & & 2-Methylnaphthalene + Fumarate $<=>2-$ & \\
\hline R06903 & reaction & Naphtylm... & 2.194E-02 \\
\hline R06987 & reaction & propanoyl-CoA:formate C-propanoyltransferase & $2.218 \mathrm{E}-02$ \\
\hline R07159 & reaction & D-glyceraldehyde-3-phosphate:ferredoxin oxido... & $1.000 \mathrm{E}-06$ \\
\hline
\end{tabular}




\begin{tabular}{|c|c|c|c|}
\hline R07274 & reaction & O-phospho-L-serine:hydrogen-sulfide 2 -amino- $2 \ldots$ & $1.948 \mathrm{E}-04$ \\
\hline R07399 & reaction & Acetyl-CoA + Pyruvate $+\mathrm{H} 2 \mathrm{O} \Leftrightarrow=>(\mathrm{R})-2-$ Methylm... & $2.986 \mathrm{E}-03$ \\
\hline R07456 & reaction & D-ribulose 5-phosphate,D-glyceraldehyde 3-pho... & $3.194 E+09$ \\
\hline R08323 & reaction & 7-Mercaptoheptanoic acid + L-Threonine + ATP ... & 5.991E-03 \\
\hline R08555 & reaction & (R)-lactate hydro-lyase (adding N-acetyl-D-gl... & $9.758 \mathrm{E}-03$ \\
\hline R08559 & reaction & protein-N(pi)-phosphohistidine:N-acetylmurama... & 1.000E-06 \\
\hline R10049 & reaction & 2-oxopropanal:D-fructose 1,6-bisphosphate gly... & 4.546E-02 \\
\hline R10052 & reaction & (2R,3S)-3-isopropylmalate:NAD+ oxidoreductase & $1.000 \mathrm{E}-06$ \\
\hline R10090 & reaction & citrate:N6-acetyl-N6-hydroxy-L-lysine ligase ... & $6.270 \mathrm{E}-03$ \\
\hline R10170 & reaction & (2R,3S)-3-isopropylmalate hydro-lyase (2-isop... & $1.000 \mathrm{E}-06$ \\
\hline R10270 & reaction & (5R)-5-phosphooxy-L-lysine phosphate-lyase (d... & 4.432E-04 \\
\hline R10343 & reaction & succinyl-CoA:acetate CoA-transferase & $1.608 \mathrm{E}-03$ \\
\hline R10660 & reaction & fumarate CoM:CoB oxidoreductase (succinate fo... & $6.353 E+09$ \\
\hline R10677 & reaction & citrate:L-glutamate ligase (ADP-forming) & $2.358 \mathrm{E}-02$ \\
\hline \multirow[t]{2}{*}{ R10699 } & reaction & L-lysine:8-amino-7-oxononanoate aminotransfer... & $1.201 \mathrm{E}-03$ \\
\hline & & D-glyceraldehyde-3-phosphate:NAD+ & \\
\hline R10860 & reaction & oxidoreduct... & $1.000 \mathrm{E}-06$ \\
\hline R10888 & reaction & C20914 + L-Threonine $<=>$ C20915 & $1.339 \mathrm{E}-02$ \\
\hline R10891 & reaction & Tabtoxin $+\mathrm{H} 2 \mathrm{O}<=>$ Tabtoxinine-beta-lactam $+\ldots$ & $1.168 \mathrm{E}-03$ \\
\hline R10892 & reaction & tabtoxinine-beta-lactam:L-threonine ligase (A... & $8.832 \mathrm{E}-04$ \\
\hline R10940 & reaction & fumarate:L-2,3-diaminopropanoate ligase (AMP-... & $6.289 \mathrm{E}-03$ \\
\hline R10973 & reaction & D-ribose-2,5-bisphosphate 2-phosphohydrolase & $2.912 \mathrm{E}-03$ \\
\hline \multirow[t]{2}{*}{ R11024 } & reaction & ATP:N-acetyl-D-muramate 1-phosphotransferase & $1.000 \mathrm{E}-06$ \\
\hline & & (S)-2-amino-6-oxohexanoate:NADP+ & \\
\hline R11679 & reaction & oxidoreducta... & $1.000 \mathrm{E}-06$ \\
\hline
\end{tabular}




\begin{tabular}{|c|c|c|c|}
\hline & & $\mathrm{N}$-acetyl-D-muramate 6-phosphate & \\
\hline R11785 & reaction & phosphohydrol... & 1.000E-06 \\
\hline R12308 & reaction & L-2,3-diaminopropanoate:citrate ligase (2-[(L... & 2.769E-03 \\
\hline R12353 & reaction & D-Ornithine + Citrate + ATP $<=>$ N5-Citryl-D-o... & 4.337E-04 \\
\hline R12354 & reaction & N5-Citryl-D-ornithine + Citrate + ATP $<=>$ Sta... & 3.695E-02 \\
\hline R12596 & reaction & Spermidine + Citrate + ATP $<=>$ N-Citryl-sperm... & $2.685 \mathrm{E}-04$ \\
\hline C00009 & compound & Orthophosphate & $9.902 \mathrm{E}-03$ \\
\hline C00042 & compound & Succinate & $1.000 \mathrm{E}-06$ \\
\hline C00058 & compound & Formate & $1.695 \mathrm{E}-02$ \\
\hline C00073 & compound & L-Methionine & $3.505 E-03$ \\
\hline C00097 & compound & L-Cysteine & $1.000 \mathrm{E}-06$ \\
\hline C00109 & compound & 2-Oxobutanoate & $1.133 \mathrm{E}-02$ \\
\hline C00117 & compound & D-Ribose 5-phosphate & $1.000 \mathrm{E}-06$ \\
\hline C00118 & compound & D-Glyceraldehyde 3-phosphate & $1.000 \mathrm{E}-06$ \\
\hline C00122 & compound & Fumarate & $1.000 \mathrm{E}-06$ \\
\hline C00141 & compound & 3-Methyl-2-oxobutanoic acid & $2.304 \mathrm{E}-02$ \\
\hline C00158 & compound & Citrate & $1.000 \mathrm{E}-06$ \\
\hline C00188 & compound & L-Threonine & $1.000 \mathrm{E}-06$ \\
\hline C00672 & compound & 2-Deoxy-D-ribose 1-phosphate & 1.000E-06 \\
\hline C00673 & compound & 2-Deoxy-D-ribose 5-phosphate & $2.442 \mathrm{E}-02$ \\
\hline C02226 & compound & 2-Methylmaleate & 3.627E-04 \\
\hline C02504 & compound & alpha-Isopropylmalate & $1.000 \mathrm{E}-06$ \\
\hline C02631 & compound & 2-Isopropylmaleate & $1.000 \mathrm{E}-06$ \\
\hline C02713 & compound & N-Acetylmuramate & $1.000 \mathrm{E}-06$ \\
\hline C03145 & compound & $\mathrm{N}$-Formylmethionine & 1.000E-06 \\
\hline
\end{tabular}


bioRxiv preprint doi: https://doi.org/10.1101/2020.09.02.280495; this version posted September 3, 2020. The copyright holder for this preprint (which was not certified by peer review) is the author/funder, who has granted bioRxiv a license to display the preprint in perpetuity. It is made available under aCC-BY-ND 4.0 International license.

\begin{tabular}{lllr|}
\hline C04076 & compound & L-2-Aminoadipate 6-semialdehyde & $1.000 \mathrm{E}-06$ \\
\hline C04411 & compound & $(2 \mathrm{R}, 3 \mathrm{~S})$-3-Isopropylmalate & $1.000 \mathrm{E}-06$ \\
\hline C06032 & compound & D-erythro-3-Methylmalate & $1.000 \mathrm{E}-06$ \\
\hline C16698 & compound & N-Acetylmuramic acid 6-phosphate & $2.110 \mathrm{E}-03$ \\
\hline
\end{tabular}

